\title{
Bounds of Fractional Metric Dimension and Applications with Grid-Related Networks
}

\author{
Ali H. Alkhaldi ${ }^{1}$, Muhammad Kamran Aslam ${ }^{2}$, Muhammad Javaid ${ }^{2, *}\left(\mathbb{D}\right.$ and Abdulaziz Mohammed Alanazi ${ }^{3}$ \\ 1 Department of Mathematics, College of Science, King Khalid University, Abha P.O. Box 9004, Saudi Arabia; \\ ahalkhaldi@kku.edu.sa \\ 2 Department of Mathematics, School of Science, University of Management and Technology, \\ Lahore 54770, Pakistan; f2018265007@umt.edu.pk \\ 3 Department of Mathematics, University of Tabuk, Tabuk 71491, Saudi Arabia; am.alenezi@ut.edu.sa \\ * Correspondence: javaidmath@gmail.com or muhammad.javaid@umt.edu.pk
}

Citation: Alkhaldi, A.H.; Aslam, M.K.; Javaid, M.; Alanazi, A.M. Bounds of Fractional Metric Dimension and Applications with Grid-Related Networks. Mathematics 2021, 9, 1383. https://doi.org/ $10.3390 /$ math 9121383

Academic Editors: Ismael González Yero and Dorota Kuziak

Received: 5 April 2021

Accepted: 10 June 2021

Published: 15 June 2021

Publisher's Note: MDPI stays neutral with regard to jurisdictional claims in published maps and institutional affiliations.

Copyright: (C) 2021 by the authors. Licensee MDPI, Basel, Switzerland. This article is an open access article distributed under the terms and conditions of the Creative Commons Attribution (CC BY) license (https:// creativecommons.org/licenses/by/ $4.0 /)$.

\begin{abstract}
Metric dimension of networks is a distance based parameter that is used to rectify the distance related problems in robotics, navigation and chemical strata. The fractional metric dimension is the latest developed weighted version of metric dimension and a generalization of the concept of local fractional metric dimension. Computing the fractional metric dimension for all the connected networks is an NP-hard problem. In this note, we find the sharp bounds of the fractional metric dimensions of all the connected networks under certain conditions. Moreover, we have calculated the fractional metric dimension of grid-like networks, called triangular and polaroid grids, with the aid of the aforementioned criteria. Moreover, we analyse the bounded and unboundedness of the fractional metric dimensions of the aforesaid networks with the help of $2 \mathrm{D}$ as well as $3 \mathrm{D}$ plots.
\end{abstract}

Keywords: connected networks; metric dimension; fractional metric dimension; resolving neighbourhoods

MSC: 05C12; 05C90; 05C15; 05C62

\section{Introduction}

Every new day brings with it developments in the fields of chemical, information and biometric sciences. With these developments, many new fields have emerged such as cheminformatics, artificial intelligence and image processing. It is pertinent to mention here that metric dimensions of networks are one of the stakeholders in their development. Consider robotics, for instance, in which we have to assign different robots to different landmarks, keeping in view economical operation costs and the least number of robots. Here, the concept of metric dimension plays a vital role in converting the current scenario into a graph theoretic version. For more details, see [1-3].

Similarly, if we look into the various disciplines of chemical sciences, such as drug discovery, bond formation in chemical compounds and the development of different kits for the diagnosis of different diseases, they are wholly indebted to the concept of distance based dimensions. In this strata, the ions, atoms or molecules are considered to be nodes, and the bonds between them are known as links. In this way, chemists, pharmacists and pathologists are able to develop drugs, chemical compounds and testing kits, which are not only accurate but also have the highest form of parsimony. To find more information on these topics, we refer to [4-6].

In a network $\mathbb{G}$, the distance $d(u, v)$ of two distinct vertices $u$ and $v$ is the length of the shortest path between them. For a subset $\mathbb{Y}=\left\{y_{1}, y_{2}, y_{3}, \ldots, y_{k}\right\} \subseteq V(\mathbb{G})$ and a node $x \in V(\mathbb{G})$, the metric form of $x$ with respect to $\mathbb{Y}$ is an ordered $k$-tuple $r(x \mid Y)=$ $\left(d\left(x, y_{1}\right), d\left(x, y_{2}\right), d\left(x, y_{3}\right), \ldots, d\left(x, y_{k}\right)\right)$. The set $\mathbb{Y}$ becomes a resolving set if each pair of distinct nodes of $\mathbb{G}$ has distinct metric forms. The resolving set with the minimum number of elements forms the metric basis for $\mathbb{G}$ and its cardinality is called the metric dimension of $\mathbb{G}$. 
Ever since Slater [1,7] and Harary and Melter [8] individually discovered the terminologies of resolving sets and related notions of the metric dimension of networks, many researchers have computed the same for different classes of networks. The results regarding the metric dimensions of path, cycle, Petersen, generalized Petersen networks and generalized Petersen multigraphs can be seen in [9-13]. Similarly, the results for the partition dimensions of convex polytopes and a hexagonal Möbius Ladder have been found in [14-16]. Moreover, the metric dimension was used by Chartrand et al. to solve the integer programming problem (IPP) [12]. Later on, Currie and Oellermann pioneered the concept of the fractional metric dimension (FMD), through which they solved the IPP for its precise results [17]. Arumugam and Mathew [18] provided the formal definition of FMD after coming across its hidden properties. Since then, many researchers have tried their luck in this area by attacking different networks that are constructed under various operations of Cartesian, hierarchical, corona, lexicographic and comb products (see [19-23]). Recently, Liu et al. [24] calculated the FMD of the generalized Jahangir network.

In this note, we are going to present the generic bounds for the FMD of connected networks. As a consequence of the obtained results, we have calculated sharp bounds for the FMDs of grid-like networks that are triangular and polaroid grids. The bounded and unboundedness of the FMDs of the same are also analysed numerically as well as graphically. This note is sequenced in the following way: Section 1 is the introduction; Section 2 sets out the preliminaries; Section 3 presents the generic criteria for the FMD of connected networks. The resolving neighbourhoods of the networks under consideration are dealt with in Section 4. In Section 5, we calculate the FMDs of $\mathbb{T}^{n}$ and $\mathbb{P} G(m, n)$. Section 6 closes this note with the conclusions of our findings by graphical and numerical analysis.

\section{Preliminaries}

Assume that $c \in V(\mathbb{G})$ and $\{a, b\} \subseteq V(\mathbb{G})$, then $\{a, b\}$ is resolved by $c$ if $d(a, c) \neq$ $d(b, c)$. The set formed by all the elements bearing the aforementioned property is known as the resolving neighbourhood. The resolving neighbourhood (RN) of $\{a, b\}$ is mathematically given by $R\{a, b\}=\{c \in V(\mathbb{G}) \mid d(a, c) \neq d(b, c)\}$. For a connected network $\mathbb{G}(V(\mathbb{G}), E(\mathbb{G}))$ of order $p$, a function $\tau: V(\mathbb{G}) \rightarrow[0,1]$ is a known resolving function (RF) of $\mathbb{G}$ if $\tau(R\{a, b\}) \geq 1 \forall$ is the resolving neighbourhoods of each pair of vertices $a, b \in V(\mathbb{G})$, where $\tau(R\{a, b\})=\sum_{c \in R\{a, b\}} \tau(c)$. An RF $\tau$ of $\mathbb{G}$ is known as a minimal resolving function (MRF) if there exists any function $\eta: V(\mathbb{G}) \rightarrow[0,1]$ such that $\eta \leq \tau$ and $\tau(c) \neq \eta(c)$ for at least one $c \in V(\mathbb{G})$ that is not an RF of $\mathbb{G}$. The FMD of a network $\mathbb{G}$ is given by $f \operatorname{dim}(\mathbb{G})=\min \{|\tau|: \tau$ is the MRF of $\mathbb{G}\}$, where $|\tau|=\sum_{c \in V(\mathbb{G})} \tau(c)$. For more details, see [18].

\section{Construction of Graphs}

The current part of the section focuses on the construction of grid-like networks.

For $1 \leq i \leq n, P_{i}$ is the paths of lengths $0,1, \ldots, n-1$. The triangular grid is a network formed by joining the first and last node of each of the two paths and the inner nodes of the first with the inner node of each of the two paths. Its order is $\frac{n(n+1)}{2}$ and its size is $\frac{3 n(n-1)}{2}$. The vertex and edge sets are given by $V\left(\mathbb{T}^{n}\right)=\bigcup_{i=1}^{n}\left\{v_{j}^{i} \mid 1 \leq j \leq i\right\}$ and

Figure 1.

$$
E\left(\mathbb{T}^{n}\right)=\bigcup_{i=1}^{n}\left\{v_{j}^{i} v_{j+1}^{i}\right\} \cup \bigcup_{i=1}^{n}\left\{v_{j}^{i} v_{j}^{i+1}\right\}, \text { respectively. The triangular grid is illustrated in }
$$




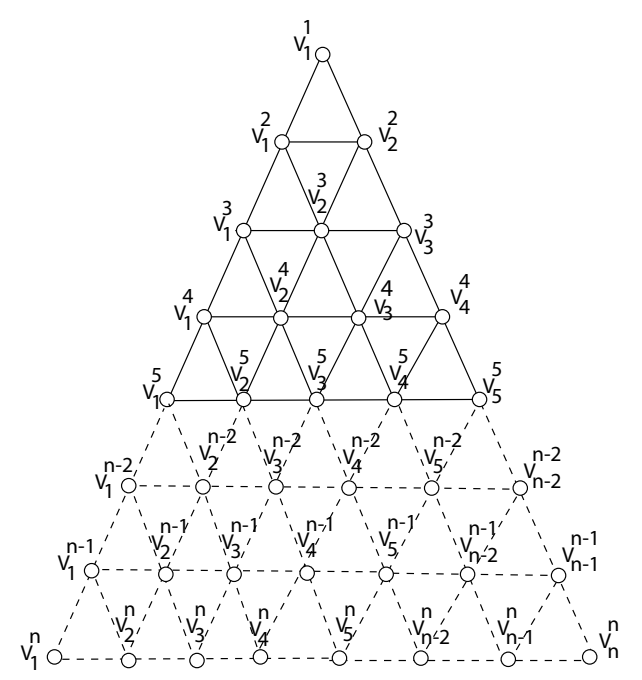

Figure 1. Triangular Grid $\mathbb{T}^{n}$.

The generalized prism is formed by the Cartesian product of $C_{m} \times P_{n}$ such that there are $n$ cycles of length $m$. The polaroid grid is a network $\mathbb{G} \cong \mathbb{P} \mathbb{G}(m, n)$, formed out of the generalized prism by joining each vertex of $C_{m}$ of its first layer to a further vertex $c$ such that its vertex and edge sets are given by $V(\mathbb{P} \mathbb{G}(m, n))=\{c\} \cup \bigcup_{i=1, j=1}^{m, n}\left\{v_{j}^{i}\right\}$ and

$$
E(\mathbb{P} G(m, n))=\bigcup_{j=1}^{n}\left\{c v_{1}^{j}\right\} \cup \bigcup_{i=1, j=1}^{m, n}\left\{v_{j}^{i} v_{j+1}^{i}\right\} \cup \bigcup_{i=1, j=1}^{m, n}\left\{v_{j}^{i} v_{j}^{i+1}\right\} \text {, respectively. Its order is }
$$
$m n+1$ and its size is $2 m n$. Figure 2 shows the polaroid grid.

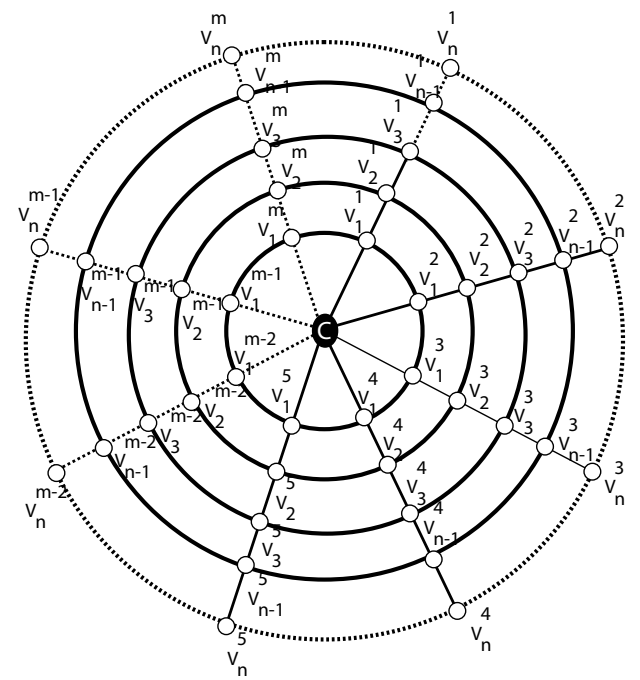

Figure 2. Polaroid Grid $\mathbb{P} \mathbb{G}(m, n)$.

\section{Sharp Bounds of FMD of Connected Networks}

Now, we are going to define the generic criteria for the FMD of connected networks as follows.

Theorem 1. Let $\mathbb{G}$ be a connected network and $R\{a, b\}$ be a resolving neighbourhood set of the pair of vertices $a, b$ in $\mathbb{G}$. If $\kappa=\min \{|R\{a, b\}|\}, X=\cup\{R\{a, b\}:|R\{a, b\}|=\kappa\}$ and $|R\{a, b\} \cap X| \geq \kappa$ then :

$$
1 \leq f \operatorname{dim}(\mathbb{G}) \leq \frac{|X|}{\kappa}
$$

where $2 \leq \kappa \leq|X|$ 
Proof. The proof of the current statement will be completed using the subsequent two cases (Case A and Case B), depending on the values of $\kappa$.

Case A: Assume that $R=R\{a, b\}, \kappa \neq V(\mathbb{G})$ and define the function $\psi: V(\mathbb{G}) \rightarrow[0,1]$ as follows:

$$
\psi(v)= \begin{cases}\frac{1}{\kappa}, & v \in X \\ 0, & v \in V(\mathbb{G})-X .\end{cases}
$$

Since $\forall a, b \in V(\mathbb{G})$,

$$
\begin{aligned}
\psi(R\{a, b\}) & =\sum_{x \in R\{a, b\}} \psi(x) \\
& =\sum_{x \in R\{a, b\} \cap X} \frac{1}{\mathcal{K}} \\
& =|R\{a, b\} \cap X| \frac{1}{\mathcal{K}} \\
& \geq 1 .
\end{aligned}
$$

This shows that $\psi$ is a resolving function. To check whether $\psi$ is a minimal resolving function, let us consider another resolving function $\tau$, such that $\tau \leq \psi$. By definition, $\tau(x)<\psi(x)$ for some $x \in X$. Assume $x \in R$ and for some $a, b \in V(\mathbb{G})$ with $|R|=\kappa$. Then

$$
\tau(R)=\sum_{x \in R} \tau(x)<\sum_{x \in R} \psi(x)<1,
$$

$\Rightarrow \tau(R)<1$, which is a contradiction. Hence, our supposition is wrong that $\tau$ is a resolving function. Thus, $\psi$ is a minimal resolving function. Now, we consider another minimal resolving function, say $\bar{\psi}$ of $\mathbb{G}$. We move further by addressing the following sub cases.

Case I: If $\bar{\psi}(R)<\frac{1}{\kappa} \forall x \in X$ : As we can see that $|R|=\kappa$ for $R \subseteq X$ thus $\bar{\psi}<\frac{1}{\kappa}$. This shows that $\bar{\psi}$ is not a resolving function. Therefore, this case does not hold.

Case II: If $\bar{\psi}(R) \geq \frac{1}{\kappa} \forall x \in X$ : In this case, we arrive at the following expression:

$$
\begin{aligned}
|\bar{\psi}| & =\sum_{x \in V(\mathbb{G})} \bar{\psi}(x)=\sum_{x \in V(\mathbb{G})-X} \bar{\psi}(x)+\sum_{x \in X} \bar{\psi}(x)>\sum_{x \in V(\mathbb{G})-X} \psi(x)+\sum_{x \in X} \psi(x) \\
& =\frac{|X|}{\kappa}=|\psi| .
\end{aligned}
$$

Finally, we have

$$
\operatorname{fim}(\mathbb{G})=\frac{|X|}{\kappa} .
$$

Case III: If $\bar{\psi}<\frac{1}{\kappa}$ for some $x \in X$ : Suppose that $T=\left\{y \in X \mid \bar{\psi}(y)<\frac{1}{\kappa}\right\}$ (where $T \subset X$ else it will be the same as Case I, which does not hold). Moreover, for $|R|=\kappa$, if $R \subseteq T$ then $\bar{\psi}(R)<1$, which is not possible as $\bar{\psi}$ will not be a resolving function. As an aftermath, for at least one $R$ in $X, T \cap R \neq \Phi$ and necessarily $|T \cap R|<|R|$ for all such resolving neighbourhoods in $\mathbb{G}$ in $X$. Let $\bar{X}=\bigcup\{R \mid T \cap R \neq \Phi\}$. Assume that:

$$
\sum_{x \in R} \bar{\psi}(x)=\sum_{x \in R \wedge x \in T} \bar{\psi}(x)+\sum_{x \in R \wedge x \in \bar{X}-T} \bar{\psi}(x) .
$$

(i) For $1 \leq r, s \leq w, R_{r} \cap R_{s}=\Phi$. 
In this case, all the resolving neighbourhoods are pairwise disjoint, thus we arrive at the following situation below:

$$
\begin{aligned}
|\bar{\psi}| & =\sum_{x \in Z} \bar{\psi}(x)+\sum_{x \in X-Z} \bar{\psi}(x) \\
& \geq \sum_{x \in Z} \psi(x)+\sum_{x \in X-Z} \psi(x) \\
& =|\psi| .
\end{aligned}
$$

Hence,

$$
f \operatorname{dim}(\mathbb{G})=\frac{|X|}{\kappa} .
$$

(ii) For $1 \leq r, s \leq w, R_{r} \cap R_{s} \neq \Phi$.

This case will give rise to the further two sub cases, discussed below: Subcase (1): $\cap R=\phi$.

As an aftermath of the present case, the further two cases need to be addressed as follows:

(a) The intersection $R_{r} \cap R_{s}$ moves in cyclic order.

For the current case, the minimum resolving neighbourhoods exhibit the behaviour of cyclic intersection, as shown in Figure 3.

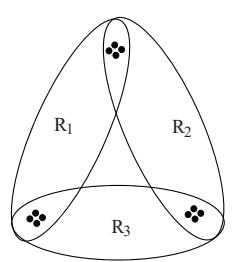

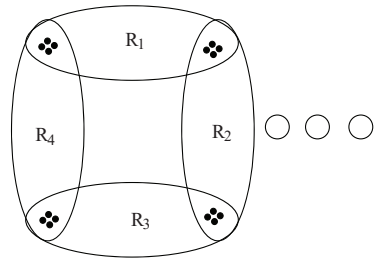

(b)

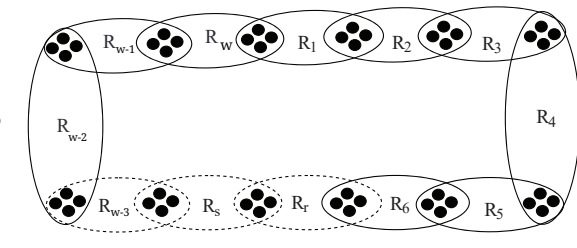

(c)

Figure 3. Cyclic order of intersection of Resolving Neighbourhoods with (a) three RNS, (b) Four RNS and (c) w RNS.

All these situations are summarized below: For $1 \leq r, s \leq w$, shown in Figure $3, \bar{\psi}$ takes the following form:

$$
\begin{gathered}
|\bar{\psi}|=\sum_{x \in X} \bar{\psi}(x)=\sum_{x \in R_{1} \cap R_{2}} \bar{\psi}(x)+\sum_{x \in R_{2} \cap R_{3}} \bar{\psi}(x)+\ldots \\
\quad+\sum_{x \in R_{r} \cap R_{s}} \bar{\psi}(x)+\cdots+\sum_{x \in R_{1} \cap R_{w}} \bar{\psi}(x) \\
\geq \sum_{x \in X} \psi(x)=\sum_{x \in R_{1} \cap R_{2}} \psi(x)+\sum_{x \in R_{2} \cap R_{3}} \psi(x)+\ldots \\
\quad+\sum_{x \in R_{r} \cap R_{s}} \psi(x)+\cdots+\sum_{x \in R_{1} \cap R_{w}} \psi(x) \\
\geq \frac{\left|R_{1} \cap R_{2}\right|}{\kappa}+\frac{\left|R_{2} \cap R_{3}\right|}{\kappa}+\cdots+\frac{\left|R_{r} \cap R_{s}\right|}{\kappa}+\cdots+\frac{\left|R_{1} \cap R_{w}\right|}{\kappa} \\
\geq \frac{\left|R_{1} \cap R_{2}\right|}{\kappa}+\frac{\left|R_{2} \cap R_{3}\right|}{\kappa}+\cdots+\frac{\left|R_{r} \cap R_{s}\right|}{\kappa}+\cdots+\frac{\left|R_{1} \cap R_{w}\right|}{\kappa} \\
\geq \frac{\left|R_{1} \cap R_{2}\right|+\left|R_{2} \cap R_{3}\right|+\cdots+\left|R_{r} \cap R_{s}\right|+\cdots+\left|R_{w} \cap R_{1}\right|}{\kappa} \\
\geq \frac{|X|}{\kappa}=|\psi| .
\end{gathered}
$$


Therefore, we have

$$
\operatorname{fim}(\mathbb{G})=\frac{|X|}{\kappa} .
$$

(b) The intersection $R_{r} \cap R_{S}$ does not move in cyclic order.

In this case, the minimum resolving neighbourhood will show the intersection that is not moving in a cyclic manner. Thus, each of them will show the dual behaviour of either the chain of subset or the non-chain of subset, but overall they do not have any element in common. So $|\bar{\psi}|$ in each of these cases takes the form as below:

$$
|\bar{\psi}| \leq \frac{|X|}{\kappa}=|\psi|
$$

Therefore, we have,

$$
\operatorname{fim}(\mathbb{G}) \leq \frac{|X|}{\kappa}
$$

Subcase (2): $\bigcap R \neq \Phi$ say $\bigcap R=Y$.

In the present case, two situations will arise as a result. The situations are:

(a) $R_{r}-Y \cap R_{s}-Y=\Phi$.

(b) $\quad R_{r}-Y \cap R_{S}-Y \neq \Phi$.

In the former one, for $1 \leq r, s \leq w$, each of $R_{r}$ and $R_{s}$ contains nothing in common except the elements of $Y$ and becomes pairwise disjoint, taking their difference from $Y$. In the latter case, $R_{r}$ and $R_{S}$ contain elements in common other than those in $Y$. Therefore, in both situations $\psi$ takes the following form:

$$
\begin{aligned}
|\bar{\psi}|= & \sum_{x \in X} \bar{\psi}(x) \leq \sum_{x \in R_{r}-Y} \bar{\psi}(x)+\sum_{x \in R_{s}-Y} \bar{\psi}(x)+\cdots+ \\
& \sum_{x \in R_{s}-Y} \bar{\psi}(x)+\cdots+\sum_{x \in R_{w}-Y} \bar{\psi}(x)+\sum_{x \in Y} \bar{\psi}(x) \\
|\psi|= & \sum_{x \in X} \psi(x) \leq \sum_{x \in R_{r}-Y} \psi(x)+\sum_{x \in R_{s}-Y} \psi(x)+\cdots+ \\
& \sum_{x \in R_{s}-Y} \psi(x)+\cdots+\sum_{x \in R_{w}-Y} \psi(x)+\sum_{x \in Y} \psi(x) \\
= & \frac{\left|R_{1}-Y\right|}{\kappa}+\frac{\left|R_{2}-Y\right|}{\kappa}+\cdots+\frac{\left|R_{r}-Y\right|}{\kappa}+ \\
& \frac{\left|R_{s}-Y\right|}{\kappa}+\cdots+\frac{\left|R_{w}-Y\right|}{\kappa}+\frac{|Y|}{\kappa} \\
= & \frac{|X-Y|+|Y|}{\kappa} \\
= & \frac{|X-Y|+|Y|}{\kappa}=\frac{|X|}{\kappa} .
\end{aligned}
$$

Therefore, we have

$$
\operatorname{fim}(\mathbb{G}) \leq \frac{|X|}{\kappa}
$$

Case V: For Some $R_{r} \cap R_{S}=\Phi$.

In this case, $X$ is has two collections that are $W=\{R \mid R$ are the resolving neighbourhoods with $\left.R_{r} \cap R_{S}=\Phi\right\}$ and $Z=\left\{R \mid R\right.$ are the resolving neighbourhoods with $\left.R_{r} \cap R_{S} \neq \Phi\right\}$ respectively, where $W \subset X$ and $Z \subset X$. Thus, for $W$ and $Z,|\psi|$ takes the form: 


$$
\begin{aligned}
|\bar{\psi}| & \leq \sum_{x \in W}^{|W|} \bar{\psi}(x)+\sum_{x \in Z}^{|Z|} \bar{\psi}(x) \\
& \geq \sum_{x \in W}^{|W|} \psi(x)+\sum_{x \in Z}^{|Z|} \psi(x) \\
& \leq \frac{|W|}{\kappa}+\frac{|Z|}{\kappa} \\
& \leq \frac{|W|+|Z|}{\kappa} \\
& \leq \frac{|X|}{\kappa} .
\end{aligned}
$$

Throughout this case, we come to the following conclusion:

$$
\operatorname{fdim}(\mathbb{G}) \leq \frac{|X|}{\kappa} .
$$

Case B: When $\kappa=|V(\mathbb{G})|$ or $\kappa=|X|$.

In this case, the cardinality of each of the minimum resolving sets $R\{a, b\}$ becomes equal to $|V(\mathbb{G})|$ or $|X|$, so in this case $|\psi|$ takes the form:

$$
\operatorname{fdim}(\mathbb{G})=\frac{|X|}{|X|}=1 .
$$

Consequently, from all the cases above, we arrive at the following conclusion:

$$
1 \leq \operatorname{fim}(\mathbb{G}) \leq \frac{|X|}{\kappa} .
$$

\section{Resolving Neighbourhoods}

This section provides insight into the resolving neighbourhoods of the networks under consideration.

\subsection{Resolving Neighbourhoods of Triangular Grid}

Lemma 1. Suppose that $\mathbb{G} \cong \mathbb{T}^{n}$ is a triangular grid network, taking any non-negative number $n \geq 5$ then:

(a) For $1 \leq l \leq 3,\left|R_{l}\right|=\left|R\left\{v_{1}^{2}, v_{2}^{2}\right\}\right|=\left|R\left\{v_{1}^{n-1}, v_{2}^{n}\right\}\right|=\left|R\left\{v_{n-1}^{n-1}, v_{n-1}^{n}\right\}\right|=2(n-1)$ and $\left|\bigcup_{t=1}^{l} R_{t}\right|=3(n-1) ;$ and

(b) For $1 \leq u \leq 7,\left|R_{l}\right| \leq\left|R_{u}\right|$ and $\left|R_{u} \cap \bigcup_{l=1}^{m} R_{l}\right| \geq\left|R_{l}\right|$, for any $R N$ set $\kappa_{u}$ of $\mathbb{G}$.

Proof. The resolving neighbourhoods with the minimum cardinality are:

$R_{1}=R\left\{v_{1}^{2}, v_{2}^{2}\right\}=\left\{v_{1}^{j} \mid 2 \leq j \leq n\right\} \cup\left\{v_{j}^{j} \mid 2 \leq j \leq n\right\}$,

$R_{2}=R\left\{v_{1}^{n-1}, v_{2}^{n}\right\}=\left\{v_{1}^{j} \mid 1 \leq j \leq n-1\right\} \cup\left\{v_{j}^{n} \mid 2 \leq j \leq n\right\}$ and

$R_{3}=R\left\{v_{n-1}^{n-1}, v_{n-1}^{n}\right\}=\left\{v_{j}^{j} \mid 1 \leq j \leq n-1\right\} \cup\left\{v_{j}^{n} \mid 1 \leq j \leq n-1\right\} ，$

respectively.

It is clear from the above that $\left|R_{l}\right|=2(n-1)$ for $1 \leq l \leq 3, \bigcup_{t=1}^{l} R_{t}=\left\{v_{1}^{j} \mid 1 \leq j \leq\right.$ $n\} \cup\left\{v_{j}^{n} \mid 2 \leq j \leq n-1\right\} \cup\left\{v_{j}^{j} \mid 2 \leq j \leq n-1\right\}$ and $\left|\bigcup_{t=1}^{l} R_{t}\right|=3(n-1)$.

(b) In order to prove the required RNs, we need the following variables:

- $1 \leq r \leq m$ 
- $\quad q \geq 1$ and $q \equiv 1(\bmod 2)$

- $s \geq 2$ and $s \equiv 0(\bmod 2)$.

The required RNs for $1 \leq u \leq 9$ are given by:

Table 1. $\dot{R}_{1}=R\left\{v_{p}^{r}, v_{p+q}^{r}\right\}$.

\section{Cases Elements}

$q=1 \quad V(\mathbb{G})-\left\{v_{k}^{j} \mid j \equiv 1,2, \ldots, r-p(\bmod n) \wedge 1 \leq k \leq j\right\} \cup\left\{v_{k}^{j} \mid r-p+1 \leq j \leq r-1 \wedge j-r+p+1 \leq k \leq\right.$ $p\} \cup\left\{v_{k}^{j} \mid r+1 \leq j \leq n \wedge p+1 \leq k \leq j-r+p\right\}$

$q \geq 3 \quad V(\mathbb{G})-\left\{v_{k}^{j} \mid j \equiv 1,2, \ldots, r-p-q+1(\bmod n) \wedge 1 \leq k \leq p \vee 1 \leq k \leq j\right\} \cup\left\{v_{k}^{j} \mid r-p-q+2 \leq j \leq r-q \wedge j-\right.$ $1 \leq k \leq p\} \cup\left\{v_{k}^{r-j} \mid j \equiv 1,3,5, \ldots, q-2(\bmod q) \wedge p+\frac{q-1}{2} \leq k \leq p+\frac{q-j}{2}\right\} \cup\left\{v_{k}^{r+j} \mid j \equiv 1,3,5, \ldots, q-2(\bmod \right.$ q) $\left.\wedge p+\frac{q+1}{2} \leq k \leq p+\frac{q+j}{2}\right\} \cup\left\{v_{k}^{j} \mid r+q \leq j \leq n \wedge p+q \leq k \leq j-r+p\right\}$

Table 2. $\dot{R}_{2}=R\left\{v_{p}^{r}, v_{p+s}^{r}\right\}$.

\begin{tabular}{ll}
\hline Cases & Elements \\
\hline$s=2$ & $V(\mathbb{G})-\left\{v_{k}^{j} \mid j \equiv 1,2, \ldots, r-p-1(\bmod n) \wedge 1 \leq k \leq j\right\} \cup\left\{v_{k}^{j} \mid r-p \leq j \leq r-2 \wedge j-2 \leq k \leq p \vee j-2 \leq\right.$ \\
& $k \leq j\} \cup\left\{v_{k}^{r} \mid k=p+1\right\} \cup\left\{v_{k}^{j} \mid r+2 \leq j \leq n \wedge p+2 \leq k \leq j-r+p\right\}$ \\
\hline$s \geq 4$ & $V(\mathbb{G})-\left\{v_{k}^{j} \mid j \equiv 1,2, \ldots, r-p-s+1(\bmod n) \wedge 1 \leq k \leq j \vee 1 \leq k \leq p\right\} \cup\left\{v_{k}^{j} \mid r-p \leq j \leq r-s \wedge j-2 \leq\right.$ \\
& $k \leq p\} \cup\left\{v_{k}^{r} \mid k=p+\frac{s}{2}\right\}\left\{v_{k}^{j} \mid r+s \leq j \leq n \wedge p+s \leq k \leq j-r+p\right\}$ \\
\hline
\end{tabular}

Table 3. $\dot{R}_{3}=R\left\{v_{p}^{r}, v_{p}^{r+q}\right\}$.

\begin{tabular}{ll}
\hline Cases & Elements \\
\hline$q=1, p=1$ & $V(\mathbb{G})-\left\{v_{k}^{j} \mid r+1 \leq j \leq n \wedge j-r+1 \leq k \leq j\right\}$ \\
\hline$q \geq 3, p=1$ & $V(\mathbb{G})-\left\{v_{k}^{j} \mid r+q-1 \leq j \leq r+q-\frac{q-1}{2} \wedge k \equiv q-1, q-2, q-3, \ldots, \frac{q+1}{2}(\bmod q)\right\} \cup\left\{v_{k}^{j} \mid r+q \leq j \leq\right.$ \\
& $n \wedge j-r+1 \leq k \leq j\}$ \\
\hline$q \geq 3, q \leq p$ & $V(\mathbb{G})-\left\{v_{k}^{j} \mid j=r+\frac{q-1}{2} \wedge p-1\right\} \cup\left\{v_{k}^{j} \mid r+\frac{q-1}{2} \leq j \leq r+\frac{q+t}{2}, t \equiv 1,3,5, \ldots, q-2(\bmod q) \wedge p+1 \leq\right.$ \\
& $k \leq p+t\} \cup\left\{v_{k}^{j} \mid r+q \leq j \leq n \wedge j-r+p \leq k \leq j\right\}$ \\
\hline$q \geq 3, p<q$ & $V(\mathbb{G})-\left\{v_{k}^{j} \mid r-p+q+1 \leq j \leq r \wedge 1 \leq k \leq j-r+p-q\right\} \cup\left\{v_{k}^{j} \mid j=r+\frac{q-1}{2} \wedge p-1\right\} \cup\left\{v_{k}^{j} \mid r+\frac{q-1}{2} \leq\right.$ \\
& $\left.j \leq r+\frac{q+t}{2}, t \equiv 1,3,5, \ldots, q-2(\bmod q) \wedge p-1 \leq k \leq p+t\right\} \cup\left\{v_{k}^{j} \mid r+q \leq j \leq n \wedge j-r+p \leq k \leq j\right\}$ \\
\hline
\end{tabular}

By symmetry, we can see that $\left|R\left\{v_{p}^{r}, v_{p}^{r+q}\right\}\right|=\left|K_{4}\right|=\left|R\left\{v_{r}^{r-p+1}, v_{r}^{r+q-p+1}\right\}\right|$. 
Table 4. $\dot{R}_{5}=R\left\{v_{p}^{r}, v_{p}^{r+s}\right\}$.

\begin{tabular}{ll}
\hline Cases & Elements \\
\hline$s=2, p=1$ & $V(\mathbb{G})-\left\{v_{1}^{j} \mid j=r+1\right\} \cup\left\{v_{k}^{j} \mid r+2 \leq j \leq n \wedge j-r+1 \leq k \leq j\right\}$ \\
\hline$s \geq 4, p=1$ & $V(\mathbb{G})-\left\{v_{1}^{j} \mid j=r+\frac{s}{2}\right\} \cup\left\{v_{k}^{j} \mid r+\frac{s+2}{2} \leq j \leq r+\frac{s+t}{2}, r \equiv 2,4,6, \ldots, s-2(\bmod s) \wedge p+2 \leq k \leq\right.$ \\
& $p+t\} \cup\left\{v_{k}^{j} \mid r+s \leq j \leq n \wedge j-r+p \leq k \leq j\right\}$ \\
\hline$s \geq 4, s \leq p$ & $V(\mathbb{G})-\left\{v_{p}^{j} \mid j=r+\frac{s}{2} \wedge\right\} \cup\left\{v_{k}^{j} \mid r+\frac{s+2}{2} \leq j \leq r+\frac{s+t}{2}, t \equiv 2,4, \ldots, s-2(\bmod s) \wedge p+2 \leq k \leq\right.$ \\
& $p+t\} \cup\left\{v_{k}^{j} \mid r+s \leq j \leq n \wedge j-s+1 \leq k \leq j\right\}$ \\
\hline$s \geq 3, p<s$ & $V(\mathbb{G})-V(\mathbb{G})-\left\{v_{k}^{j} \mid r-p+s+1 \leq j \leq r \wedge 1 \leq k \leq j-r+s-1\right\} \cup\left\{v_{p}^{j} \mid j=r+\frac{s}{2} \wedge\right\} \cup\left\{v_{k}^{j} \mid r+\frac{s+2}{2} \leq\right.$ \\
& $\left.j \leq r+\frac{s+t}{2}, t \equiv 2,4, \ldots, s-2(\bmod s) \wedge p+2 \leq k \leq p+t\right\} \cup\left\{v_{k}^{j} \mid r+s \leq j \leq n \wedge j-s+1 \leq k \leq j\right\}$ \\
\hline
\end{tabular}

By symmetry, we can see that $\left|R\left\{v_{p}^{r}, v_{p}^{r+s}\right\}\right|=\left|\hat{R}_{6}\right|=\left|R\left\{v_{r}^{r-s+1}, v_{r}^{r+s-p+1}\right\}\right|$.

Table 5. $\hat{R}_{u}$ for $7 \leq u \leq 9$.

\begin{tabular}{ll}
\hline Cases & Elements \\
\hline$R\left\{v_{p}^{r}, v_{p+q}^{r+s}\right\}$ & $V(\mathbb{G})-\left\{v_{j}^{k} \mid k=r+\frac{s}{2} \wedge 1 \leq j \leq k\right\}$ \\
$R\left\{v_{p}^{r}, v_{p+q}^{r+s}\right\}$ & $V(\mathbb{G})-\left\{v_{k}^{j} \mid p+\frac{s}{2} \leq j \leq n \wedge k=p+\frac{s}{2}\right\}$ \\
$R\left\{v_{p}^{r}, v_{p-q}^{r+q}\right\}$ & $V(\mathbb{G})-\left\{v_{k}^{j} \mid r \leq j \leq n \wedge p-q \leq k \leq n-q\right\}$ \\
\hline
\end{tabular}

Due to the symmetry of the network, it can be seen that $\left|R\left\{v_{p}^{r}, v_{p+q}^{r+s}\right\}\right|=\left|\hat{R}_{9}\right|=$ $\left|R\left\{v_{p}^{r}, v_{p-q}^{r+q}\right\}\right|$. follows:

The cardinalities of $\dot{R}_{u}$ for $1 \leq u \leq 9$, as seen in Tables $1-5$, can be summarized as

- $\left|\hat{R}_{1}\right|=\frac{1}{2} n(n+1)-2 p(r-p-q+1)-2(q-1)-(n-r-q+1)(n-r-q+2)$,

- $\left|\hat{R}_{2}\right|=\frac{1}{2} n(n+1)-2 p(r-p-s+1)-(n-r-s+1)(n-r-s+2)-2(s-1)$,

$\begin{aligned} & \text { - }\left|\hat{R}_{3}\right|=\left|\hat{R}_{4}\right|= \begin{cases}\frac{n(n+1)-p(p-1)-2(r-p+1)(n-r)}{2} & \text { for } q=1, \\ \frac{n(n+1)-(q-1)-2(r-p+1)(n-r-q+1)}{2} & \text { for } q \geq 3 \wedge p=1 \vee p=r-1, \\ \frac{n(n+1)-(p-q)(p-q+1)-(q+1)-2(r-p+1)(n-r-q+1)}{2} & \text { for } q \geq 3 \wedge 2 \leq p<r-q,\end{cases} \\ & \text { - } \quad\left|\hat{R}_{5}\right|=\left|\hat{R}_{6}\right|= \begin{cases}\frac{n(n+1)-(p-1)(p-2)-2(r-p+1)(n-r-1)-2}{2} & \text { for } s=2, \\ \frac{n(n+1)-2(s-2)-2 r(n-r-s+1)}{2} & \text { for } s \geq 4 \wedge p=1 \vee p=r, \\ \frac{n(n+1)-(p-s)(p-s+1)-(s+2)-2(r-p+1)(n-r-s+1)}{2} & \text { for } s \geq 4 \wedge 2 \leq p \leq r-s,\end{cases} \end{aligned}$

- $\left|\hat{R}_{7}\right|=\left|R\left\{v_{p}^{r}, v_{p+q}^{r+s}\right\}\right|=\frac{n(n+1)-2 r-s}{2}$,

- $\left|\hat{R}_{8}\right|=\left|\hat{R}_{9}\right|=\frac{n(n+1)-2 p-s}{2}$. $\bigcup_{l=1}^{3} R_{l}|\geq| R_{l} \mid$. 


\subsection{Resolving Neighbourhoods of Polaroid Grid}

Lemma 2. Suppose that $\mathbb{G} \cong \mathbb{P G}(m, n)$ is a polaroid grid network, taking any non-negative number $m, n \geq 3$, then:

(a) For $1 \leq l, r \leq m,\left|R_{l}\right|=\left|R\left\{v_{1}^{l}, v_{1}^{l+1}\right\}\right|=4 n$ and $\left|\bigcup_{l=1}^{m} R_{l}\right|=m n$;

(b) For $1 \leq u \leq 15,\left|R_{l}\right| \leq\left|\hat{R}_{u}\right|$ and $\left|\hat{R}_{u} \cap \bigcup_{l=1}^{m} R_{l}\right| \geq\left|R_{l}\right|$, for any $R N$ set $\hat{R}_{u}$ of $\mathbb{G}$.

Proof. (a) The resolving neighbourhoods with minimum cardinality are:

$R_{1}=R\left\{v_{1}^{r}, v_{1}^{r+1}\right\}=V(\mathbb{G})-\{c\} \cup\left\{v_{k}^{j} \mid j \equiv r+3, r+4, \ldots, r+m-2(\bmod m) \wedge 1 \leq k \leq n\right\}$.

We can see from the above that $\left|R_{l}\right|=4 n, \bigcup_{l=1}^{m} R_{l}=\bigcup_{r=1}^{m} R_{r}=V(\mathbb{G})-\{c\}$ and $=\left|\bigcup_{l=1}^{m} R_{l}\right|=\left|\bigcup_{r=1}^{m} R_{r}\right|=m n$.

(b) We define the following variables to define the required $\mathrm{RN}$ sets:

- $1 \leq r \leq m$ and $1 \leq p \leq n$

- $\quad q \geq 1$ and $q \equiv 1(\bmod 2)$

- $\quad s \geq 2$ and $s \equiv 0(\bmod 2)$.

The resolving neighbourhoods $\dot{R}_{u}$ for $1 \leq u \leq 17$ are given by: $\dot{R}_{1}=R\left\{v_{p}^{r}, v_{p+q}^{r}\right\}=\dot{R}_{2}=$ $R\left\{v_{p}^{r}, v_{p+q}^{r}\right\}=\dot{R}_{3}=R\left\{v_{p}^{r}, v_{p+s}^{r+q}\right\}=\dot{R}_{4}=R\left\{v_{r}^{r}, v_{p+q}^{r+s}\right\}=\dot{R}_{5}=R\left\{v_{1}^{r}, v_{2}^{r+s}\right\}=V(G)$,

$R_{6}=R\left\{v_{1}^{r}, v_{1}^{r+2}\right\}=V(\mathbb{G})-\{c\} \cup\left\{v_{k}^{j} \mid j=r+1 \wedge 1 \leq k \leq n\right\} \cup\left\{v_{k}^{j} \mid j \equiv r+4, r+5, \ldots, r+\right.$ $m-2(\bmod m) \wedge 1 \leq k \leq n\}, R_{7}=R\left\{v_{p}^{r}, v_{p+s}^{r}\right\}=V(G)-\left\{v_{k}^{j} \mid 1 \leq j \leq m \wedge p+\frac{s}{2}\right\}$,

$\hat{R}_{8}=R\left\{v_{1}^{r}, v_{1}^{r+q}\right\}=V(\mathbb{G})-\{c\} \cup\left\{v_{k}^{j} \mid j \equiv r+q+2, r+q+3, \ldots, r+q+m-2(\bmod \right.$ $m) \wedge 1 \leq k \leq n\}$,

$\hat{R}_{9}=R\left\{v_{1}^{r}, v_{1}^{r+s}\right\}=V(\mathbb{G})-\{c\} \cup\left\{v_{k}^{j} \mid j=r+\frac{s}{2} \wedge 1 \leq k \leq n\right\} \cup\left\{v_{k}^{j} \mid j \equiv r+s+2, r+s+\right.$ $3, \ldots, r+s+m-2(\bmod m) \wedge 1 \leq k \leq n\}$

$\hat{R}_{10}=R\left\{v_{p}^{r}, v_{p}^{r+q}\right\}=V(\mathbb{G})-\{c\} \cup\left\{v_{1}^{j} \mid j \equiv r+q+2, r+q+3, \ldots, r+q+m-2(\bmod m)\right\}$,

$\hat{R}_{11}=R\left\{v_{p}^{r}, v_{p}^{r+s}\right\}=V(\mathbb{G})-\{c\} \cup\left\{v_{k}^{j} \mid j=r+\frac{s}{2} \wedge 1 \leq k \leq n\right\} \cup\left\{v_{1}^{j} \mid j \equiv p+s+2, p+q+\right.$ $3, \ldots, p+q+m-2\}$,

$\hat{R}_{12}=R\left\{c v_{q}^{r}\right\}=V(\mathbb{G})-\left\{v_{k}^{j} \mid j \equiv r-\frac{q-1}{2}, r+\frac{q-1}{2}(\bmod m) \wedge k=\frac{q+1}{2}\right\}-\left\{v_{k}^{j} \mid j \equiv r-q, r+\right.$ $q(\bmod m) \wedge q \leq k \leq n\}$,

$\grave{R}_{13}=R\left\{c v_{s}^{r}\right\}=V(\mathbb{G})-\left\{v_{k}^{j} \mid j \equiv r-\frac{s}{2}, r, r+\frac{s}{2}(\bmod m) \wedge k=\frac{s+2}{2}\right\} \cup\left\{v_{k}^{j} \mid j \equiv r-s, r+\right.$ $s(\bmod m) \wedge s \leq k \leq n\} \hat{R}_{14}=R\left\{v_{p}^{r}, v_{p+1}^{r+1}\right\}=V(\mathbb{G})-\left\{v_{k}^{j} \mid j \equiv r+1, r+2(\bmod m) \wedge 1 \leq\right.$ $k \leq p\}-\left\{v_{k}^{j} \mid j \equiv r, r-1, r-2(\bmod m) \wedge 1 \leq k \leq n\right\}$ and $\hat{R}_{15}=R\left\{v_{p}^{r-1}, v_{p-1}^{r}\right\}=$ $V(\mathbb{G})-\left\{v_{k}^{j} \mid j \equiv r, r-1(\bmod m) \wedge 1 \leq k \leq p\right\}-\left\{v_{k}^{j} \mid j \equiv r, r+1, r+2(\bmod m) \wedge 1 \leq k \leq n\right\}$, respectively. Table 6 summarizes their cardinalities:

Table 6. Cardinalities of $\hat{R}_{u}$ for $1 \leq u \leq 15$ and $1 \leq j \leq 6$.

\begin{tabular}{llllll}
\hline RNs & Cardinality & RNs & Cardinality & RNs & Cardinality \\
\hline$\dot{R}_{j}$ & $|V(\mathbb{G})|$ & $\dot{R}_{6}$ & $4 n$ & $\dot{R}_{7}$ & $m(n-1)+1$ \\
\hline$\dot{R}_{8}$ & $(q+3) n$ & $\dot{R}_{9}$ & $(s+2) n$ & $\dot{R}_{10}$ & $m(n-1)-n+s+3$ \\
\hline$\dot{R}_{11}$ & $m(n-1)-n+s+3$ & $\dot{R}_{12}$ & $(m-2) n+2 p-4$ & $\dot{R}_{13}$ & $(m-2) n+2 s-5$ \\
\hline$\dot{R}_{14}$ & $(m-2) n+2 p-4$ & $\dot{R}_{15}$ & $(m-2) n+2 p-4$ & & \\
\hline
\end{tabular}

We can see that $\left|R_{l}\right|=4 n, \bigcup_{l=1}^{m} R_{l}=V(\mathbb{G})-\{c\}$ and $\left|\bigcup_{l=1}^{m} R_{l}\right|=m n$. For $1 \leq u \leq 15$, $\left|R_{u}\right| \leq\left|R_{l}\right| ;$ therefore, $\left|\hat{R}_{u} \cap \bigcup_{l=1}^{m} R_{l}\right| \geq\left|R_{l}\right|$. 


\section{FMD of Grid-Like Graphs}

In this section, we will calculate the sharp bounds for the FMDs of $\mathbb{T}^{n}$ and $\mathbb{P} \mathbb{G}(m, n)$ by applying Theorem 1 .

\subsection{FMD of Triangular Grid}

Theorem 2. Suppose that $\mathbb{G} \cong \mathbb{T}^{n}$ is a triangular grid network, taking any non-negative number $n \geq 5$, then $f \operatorname{dim}(\mathbb{G})=\frac{3}{2}$.

Proof. Case I: For $n=5$ : Using symmetry, the resolving neighbourhoods for the case under consideration are as follows:

Table 7. $R_{l}$ for $1 \leq l \leq 3$.

\begin{tabular}{ll}
\hline R. N. & Elements \\
\hline$R_{1}=R\left\{v_{1}^{2}, v_{2}^{2}\right\}$ & $\left\{v_{1}^{2}, v_{1}^{3}, v_{1}^{4}, v_{1}^{5}\right\} \cup\left\{v_{2}^{2}, v_{3}^{3}, v_{4}^{4}, v_{4}^{5}\right\}$ \\
$R_{2}=R\left\{v_{1}^{4}, v_{2}^{5}\right\}$ & $\left\{v_{1}^{1}, v_{1}^{2}, v_{1}^{3}, v_{1}^{4}\right\} \cup\left\{v_{2}^{5}, v_{3}^{5}, v_{4}^{5}, v_{5}^{5}\right\}$ \\
$R_{3}=R\left\{v_{4}^{4}, v_{4}^{5}\right\}$ & $\left\{v_{1}^{5}, v_{2}^{5}, v_{3}^{5}, v_{4}^{5}\right\} \cup\left\{v_{1}^{1}, v_{2}^{2}, v_{3}^{3}, v_{4}^{4}\right\}$ \\
\hline
\end{tabular}

Table 8. $\dot{R}_{u}$ for $1 \leq u \leq 68$.

\begin{tabular}{|c|c|c|c|}
\hline R. N. & Elements & R. N. & Elements \\
\hline$R\left\{v_{1}^{1}, v_{1}^{2}\right\}$ & $V(\mathbb{G})-\left\{v_{2}^{2}, v_{3}^{3}, v_{4}^{4}, v_{5}^{5}\right\}$ & $R\left\{v_{1}^{2}, v_{1}^{3}\right\}$ & $V(\mathbb{G})-\left\{v_{2}^{3}, v_{3}^{4}, v_{3}^{5}\right\} \cup\left\{v_{3}^{3}, v_{4}^{4}, v_{5}^{5}\right\}$ \\
\hline$R\left\{v_{1}^{3}, v_{1}^{4}\right\}$ & $V(\mathbb{G})-\left\{v_{2}^{4}, v_{3}^{5}\right\} \cup\left\{v_{3}^{4}, v_{4}^{5}\right\} \cup\left\{v_{4}^{4}, v_{5}^{5}\right\}$ & $R\left\{v_{1}^{4}, v_{1}^{5}\right\}$ & $V(\mathbb{G})-\left\{v_{2}^{5}\right\} \cup\left\{v_{3}^{5}\right\} \cup\left\{v_{4}^{5}\right\} \cup\left\{v_{5}^{5}\right\}$ \\
\hline$R\left\{v_{1}^{2}, v_{2}^{3}\right\}$ & $V(\mathbb{G})-\left\{v_{2}^{2}\right\} \cup\left\{v_{1}^{3}, v_{1}^{4}, v_{1}^{5}\right\}$ & $R\left\{v_{1}^{3}, v_{2}^{4}\right\}$ & $V(\mathbb{G})-\left\{v_{2}^{2}\right\} \cup\left\{v_{2}^{3}, v_{3}^{3}\right\} \cup\left\{v_{1}^{4}, v_{1}^{5}\right\}$ \\
\hline$R\left\{v_{1}^{1}, v_{1}^{4}\right\}$ & $V(\mathbb{G})-\left\{v_{2}^{3}\right\} \cup\left\{v_{4}^{4}, v_{5}^{5}\right\}$ & $R\left\{v_{1}^{2}, v_{1}^{5}\right\}$ & $V(\mathbb{G})-\left\{v_{2}^{4}\right\} \cup\left\{v_{4}^{5}, v_{5}^{5}\right\}$ \\
\hline$R\left\{v_{1}^{1}, v_{2}^{2}\right\}$ & $V(\mathbb{G})-\left\{v_{1}^{2}, v_{1}^{3}, v_{1}^{4}, v_{1}^{5}\right\}$ & $R\left\{v_{2}^{3}, v_{3}^{4}\right\}$ & $V(\mathbb{G})-\left\{v_{3}^{3}\right\} \cup\left\{v_{1}^{4}, v_{1}^{5}\right\} \cup\left\{v_{2}^{4}, v_{2}^{5}\right\}$ \\
\hline$R\left\{v_{3}^{4}, v_{4}^{5}\right\}$ & $V(\mathbb{G})-\left\{v_{4}^{4}\right\} \cup\left\{v_{1}^{5}, v_{2}^{5}, v_{3}^{5}\right\}$ & $R\left\{v_{2}^{4}, v_{3}^{5}\right\}$ & $V(\mathbb{G})-\left\{v_{3}^{3}\right\} \cup\left\{v_{3}^{4}, v_{4}^{4}\right\} \cup\left\{v_{1}^{5}, v_{2}^{5}\right\}$ \\
\hline$R\left\{v_{2}^{2}, v_{4}^{4}\right\}$ & $V(\mathbb{G})-\left\{v_{3}^{3}\right\} \cup\left\{v_{1}^{4}, v_{1}^{4}\right\} \cup\left\{v_{1}^{5}, v_{2}^{5}\right\}$ & $R\left\{v_{3}^{3}, v_{5}^{5}\right\}$ & $V(\mathbb{G})-\left\{v_{4}^{4}\right\} \cup\left\{v_{1}^{5}, v_{2}^{5}, v_{3}^{5}\right\}$ \\
\hline$R\left\{v_{1}^{1}, v_{5}^{5}\right\}$ & $V(\mathbb{G})-\left\{v_{3}^{3}, v_{2}^{4}\right\} \cup\left\{v_{1}^{5}\right\}$ & $R\left\{v_{1}^{3}, v_{2}^{3}\right\}$ & $V(\mathbb{G})-\left\{v_{1}^{1}, v_{1}^{2}\right\} \cup\left\{v_{2}^{4}\right\} \cup\left\{v_{2}^{5}, v_{3}^{5}\right\}$ \\
\hline$R\left\{v_{2}^{3}, v_{3}^{3}\right\}$ & $V(\mathbb{G})-\left\{v_{1}^{1}, v_{2}^{2}\right\} \cup\left\{v_{3}^{4}\right\} \cup\left\{v_{3}^{5}, v_{4}^{5}\right\}$ & $R\left\{v_{1}^{4}, v_{2}^{4}\right\}$ & $V(\mathbb{G})-\left\{v_{1}^{1}, v_{1}^{2}, v_{1}^{3}\right\} \cup\left\{v_{2}^{5}\right\}$ \\
\hline$R\left\{v_{2}^{4}, v_{3}^{4}\right\}$ & $V(\mathbb{G})-\left\{v_{1}^{1}\right\} \cup\left\{v_{2}^{3}\right\} \cup\left\{v_{3}^{5}\right\}$ & $R\left\{v_{3}^{4}, v_{4}^{4}\right\}$ & $V(\mathbb{G})-\left\{v_{1}^{1}, v_{2}^{2}, v_{3}^{3}\right\} \cup\left\{v_{4}^{5}\right\}$ \\
\hline$R\left\{v_{1}^{5}, v_{2}^{5}\right\}$ & $\left.V(\mathbb{G})-\left\{v_{1}^{1}\right\} \cup\left\{v_{1}^{2}, v_{2}^{2}\right\} \cup\left\{v_{1}^{3}, v_{2}^{3}\right\}, v_{1}^{4}\right\}$ & $R\left\{v_{2}^{5}, v_{3}^{5}\right\}$ & $V(\mathbb{G})-\left\{v_{1}^{1}, v_{1}^{2}\right\} \cup\left\{v_{1}^{3}, v_{2}^{3}\right\} \cup\left\{v_{2}^{4}\right\}$ \\
\hline$R\left\{v_{3}^{5}, v_{4}^{5}\right\}$ & $V(\mathbb{G})-\left\{v_{1}^{1}, v_{2}^{2}\right\} \cup\left\{v_{2}^{3}, v_{3}^{3}\right\} \cup\left\{v_{3}^{4}\right\}$ & $R\left\{v_{4}^{5}, v_{5}^{5}\right\}$ & $V(\mathbb{G})-\left\{v_{1}^{1}, v_{2}^{2}, v_{3}^{3}, v_{4}^{4}\right\}$ \\
\hline$R\left\{v_{1}^{3}, v_{3}^{3}\right\}$ & $V(\mathbb{G})-\left\{v_{1}^{1}, v_{2}^{3}, v_{3}^{5}\right\}$ & $R\left\{v_{1}^{4}, v_{3}^{4}\right\}$ & $V(\mathbb{G})-\left\{v_{1}^{1}, v_{1}^{2}, v_{2}^{4}\right\}$ \\
\hline$R\left\{v_{2}^{4}, v_{4}^{4}\right\}$ & $V(\mathbb{G})-\left\{v_{1}^{1}, v_{2}^{2}, v_{3}^{4}\right\}$ & $R\left\{v_{1}^{5}, v_{3}^{5}\right\}$ & $V(\mathbb{G})-\left\{v_{1}^{1}, v_{1}^{2}, v_{1}^{3}\right\} \cup\left\{v_{2}^{5}\right\}$ \\
\hline$R\left\{v_{2}^{5}, v_{4}^{5}\right\}$ & $V(\mathbb{G})-\left\{v_{1}^{1}, v_{2}^{3}, v_{3}^{5}\right\}$ & $R\left\{v_{3}^{5}, v_{5}^{5}\right\}$ & $V(\mathbb{G})-\left\{v_{1}^{1}, v_{2}^{2}, v_{3}^{3}, v_{4}^{5}\right\}$ \\
\hline$R\left\{v_{1}^{5}, v_{5}^{5}\right\}$ & $V(\mathbb{G})-\left\{v_{1}^{1}, v_{2}^{3}, v_{3}^{5}\right\}$ & $R\left\{v_{1}^{4}, v_{4}^{4}\right\}$ & $V(\mathbb{G})-\left\{v_{1}^{1}, v_{2}^{3}, v_{3}^{5}\right\}$ \\
\hline$R\left\{v_{1}^{5}, v_{4}^{5}\right\}$ & $V(\mathbb{G})-\left\{v_{1}^{1}, v_{1}^{2}, v_{2}^{4}\right\}$ & $R\left\{v_{2}^{5}, v_{5}^{5}\right\}$ & $V(\mathbb{G})-\left\{v_{1}^{1}, v_{2}^{2}, v_{3}^{4}\right\}$ \\
\hline$R\left\{v_{1}^{1}, v_{2}^{3}\right\}$ & $V(\mathbb{G})-\left\{v_{1}^{2}, v_{2}^{2}\right\}$ & $R\left\{v_{2}^{3}, v_{3}^{5}\right\}$ & $V(\mathbb{G})-\left\{v_{1}^{4}, v_{2}^{4}, v_{3}^{4}, v_{4}^{4}\right\}$ \\
\hline$R\left\{v_{1}^{2}, v_{2}^{4}\right\}$ & $V(\mathbb{G})-\left\{v_{1}^{3}, v_{2}^{3}, v_{3}^{3}\right\}$ & $R\left\{v_{2}^{2}, v_{3}^{4}\right\}$ & $V(\mathbb{G})-\left\{v_{1}^{3}, v_{2}^{3}, v_{3}^{3}\right\}$ \\
\hline$R\left\{v_{1}^{3}, v_{2}^{5}\right\}$ & $V(\mathbb{G})-\left\{v_{1}^{4}, v_{2}^{4}, v_{3}^{4}, v_{4}^{4}\right\}$ & $R\left\{v_{2}^{3}, v_{3}^{5}\right\}$ & $V(\mathbb{G})-\left\{v_{1}^{4}, v_{2}^{4}, v_{3}^{4}, v_{4}^{4}\right\}$ \\
\hline$R\left\{v_{3}^{3}, v_{4}^{5}\right\}$ & $V(\mathbb{G})-\left\{v_{1}^{4}, v_{2}^{4}, v_{3}^{4}, v_{4}^{4}\right\}$ & $R\left\{v_{1}^{1}, v_{2}^{5}\right\}$ & $V(\mathbb{G})-\left\{v_{1}^{3}, v_{2}^{3}, v_{4}^{4}\right\}$ \\
\hline
\end{tabular}


Table 8. Cont.

\begin{tabular}{llll}
\hline R. N. & Elements & R. N. & Elements \\
\hline$R\left\{v_{1}^{1}, v_{3}^{5}\right\}$ & $V(\mathbb{G})-\left\{v_{1}^{3}, v_{2}^{3}, v_{3}^{3}\right\}$ & $R\left\{v_{1}^{1}, v_{4}^{5}\right\}$ & $V(\mathbb{G})-\left\{v_{2}^{3}, v_{3}^{3}, v_{1}^{4}\right\}$ \\
\hline$R\left\{v_{1}^{2}, v_{2}^{5}\right\}$ & $V(\mathbb{G})-\left\{v_{3}^{4}, v_{4}^{4}\right\}$ & $R\left\{v_{1}^{2}, v_{3}^{5}\right\}$ & $V(\mathbb{G})-\left\{v_{3}^{3}, v_{1}^{4}\right\}$ \\
\hline$R\left\{v_{1}^{2}, v_{4}^{5}\right\}$ & $V(\mathbb{G})-\left\{v_{3}^{3}, v_{2}^{4}, v_{1}^{5}\right\}$ & $R\left\{v_{1}^{2}, v_{5}^{5}\right\}$ & $V(\mathbb{G})-\left\{v_{3}^{3}, v_{3}^{4}, v_{2}^{5}\right\}$ \\
\hline$R\left\{v_{2}^{2}, v_{1}^{5}\right\}$ & $V(\mathbb{G})-\left\{v_{1}^{3}, v_{2}^{4}, v_{4}^{5}\right\}$ & $R\left\{v_{2}^{2}, v_{2}^{5}\right\}$ & $V(\mathbb{G})-\left\{v_{1}^{3}, v_{3}^{4}, v_{5}^{5}\right\}$ \\
\hline$R\left\{v_{2}^{2}, v_{3}^{5}\right\}$ & $V(\mathbb{G})-\left\{v_{1}^{3}, v_{4}^{4}\right\}$ & $R\left\{v_{2}^{2}, v_{4}^{5}\right\}$ & $V(\mathbb{G})-\left\{v_{1}^{4}, v_{2}^{4}\right\}$ \\
\hline$R\left\{v_{2}^{2}, v_{1}^{3}\right\}$ & $V(\mathbb{G})-\left\{v_{1}^{2}, v_{2}^{3}, v_{3}^{4}, v_{4}^{5}\right\}$ & $R\left\{v_{1}^{2}, v_{3}^{3}\right\}$ & $V(\mathbb{G})-\left\{v_{2}^{2}, v_{2}^{3}, v_{2}^{4}, v_{2}^{5}\right\}$ \\
\hline$R\left\{v_{2}^{3}, v_{4}^{4}\right\}$ & $V(\mathbb{G})-\left\{v_{3}^{3}, v_{3}^{4}, v_{3}^{5}\right\}$ & $R\left\{v_{2}^{3}, v_{1}^{4}\right\}$ & $V(\mathbb{G})-\left\{v_{1}^{3}, v_{2}^{4}, v_{3}^{5}\right\}$ \\
\hline$R\left\{v_{3}^{4}, v_{5}^{5}\right\}$ & $V(\mathbb{G})-\left\{v_{4}^{4}, v_{5}^{5}\right\}$ & $R\left\{v_{3}^{4}, v_{1}^{5}\right\}$ & $V(\mathbb{G})-\left\{v_{1}^{4}, v_{2}^{4}\right\}$ \\
\hline$R\left\{v_{1}^{2}, v_{1}^{4}\right\}$ & $V(\mathbb{G})-\left\{v_{1}^{3}, v_{4}^{4}, v_{4}^{5}\right\}$ & $R\left\{v_{1}^{2}, v_{3}^{4}\right\}$ & $V(\mathbb{G})-\left\{v_{2}^{3}, v_{1}^{4}, v_{1}^{5}\right\}$ \\
\hline$R\left\{v_{1}^{2}, v_{4}^{4}\right\}$ & $V(\mathbb{G})-\left\{v_{2}^{4}, v_{2}^{4}\right\}$ & $R\left\{v_{1}^{3}, v_{3}^{5}\right\}$ & $V(\mathbb{G})-\left\{v_{3}^{3}, v_{2}^{4}, v_{1}^{5}\right\}$ \\
\hline$R\left\{v_{1}^{3}, v_{4}^{5}\right\}$ & $V(\mathbb{G})-\left\{v_{3}^{3}, v_{2}^{5}\right\}$ & $R\left\{v_{1}^{3}, v_{5}^{5}\right\}$ & $V(\mathbb{G})-\left\{v_{3}^{4}, v_{3}^{5}\right\}$ \\
\hline$R\left\{v_{2}^{3}, v_{1}^{5}\right\}$ & $V(\mathbb{G})-\left\{v_{3}^{5}\right\}$ & $R\left\{v_{2}^{3}, v_{2}^{5}\right\}$ & $V(\mathbb{G})-\left\{v_{2}^{4}, v_{4}^{5}, v_{5}^{5}\right\}$ \\
\hline$R\left\{v_{2}^{3}, v_{4}^{5}\right\}$ & $V(\mathbb{G})-\left\{v_{3}^{4}, v_{1}^{5}, v_{2}^{5}\right\}$ & $R\left\{v_{2}^{3}, v_{5}^{5}\right\}$ & $V(\mathbb{G})-\left\{v_{4}^{5}\right\}$ \\
\hline
\end{tabular}

The RNs depicted in Table 7 are of a minimum cardinality of 8 . Whereas Table 8 shows the RN sets with the maximum cardinality. Thus, $\bigcup_{l=1}^{3} R_{l}=\left\{v_{1}^{1}, v_{2}^{1}, v_{3}^{1}, v_{4}^{1}, v_{5}^{1}\right\} \cup$ $\left\{v_{2}^{2}, v_{3}^{3}, v_{4}^{4}, v_{5}^{5}\right\} \cup\left\{v_{5}^{2}, v_{5}^{3}, v_{5}^{4}\right\}$. We can see that $\left|\bigcup_{l=1}^{3} R_{l}\right|=12$ and $\left|\hat{R}_{u} \cap \bigcup_{l=1}^{3} R_{l}\right| \geq\left|R_{l}\right|$. Now, we define a mapping $\psi: V(\mathbb{G}) \rightarrow[0,1]$, such that $\tau\left(v_{k}^{j}\right)=\frac{1}{8}$. Since $R_{l}$ for $1 \leq l \leq 3$ exhibits a cyclic intersection with $\bigcap_{l=1}^{3} R_{l}=\phi$, by Theorem 1, Case A, Sub case(1)(a), we have $f \operatorname{dim}(\mathbb{G})=\sum_{l=1}^{3} \frac{1}{8} \leq \frac{12}{8}=\frac{3}{2}$.

Case II: For any $n \geq 6$ : Lemma 1 confirms that the minimum resolving sets are $R_{1}=$ $R\left\{v_{1}^{2}, v_{2}^{2}\right\}, R_{2}=R\left\{v_{1}^{n-1}, v_{2}^{n}\right\}$ and $R_{3}=R\left\{v_{n-1}^{n-1}, v_{n-1}^{n}\right\}$, respectively. The same affirms that $\left|R_{l}\right|=2(n-1)$ and $\left|\bigcup_{t=1}^{2} R_{t}\right|=3(n-1)$ and $\left|R\{a, b\} \cap \bigcup_{t=1}^{3} R_{t}\right| \geq\left|R_{l}\right| \forall\{a, b\} \in V(\mathbb{G})$. Let $\sigma=\left|\bigcup_{l=1}^{3} R_{l}\right|=3(n-1)$ and $\kappa=\left|R_{l}\right|=2(n-1)$. Then, we define a mapping $\tau: V(\mathbb{G}) \rightarrow[0,1]$, such that

$$
\tau(v)= \begin{cases}\frac{1}{\kappa} & \text { for } a \in \bigcup_{l=1}^{3} R_{t}, \\ 0 & \text { for } a \in V(\mathbb{C})-\bigcup_{l=1}^{3} R_{t} .\end{cases}
$$

It can be seen that $\tau$ is a resolving function for $\mathbb{G}$ with $n \geq 3$ because $\tau(R\{a, b\}) \geq 1 \forall$ $u, v \in V(\mathbb{G})$. On the contrary, assume that there is another resolving function $\rho$, such that $\rho(u) \leq \tau(u)$, for at least one $u \in V(\mathbb{G}) \rho(u) \neq \tau(u)$. As a consequence, $\rho(R\{a, b\})<1$, where $R\{a, b\}$ is a resolving neighbourhood of $\mathbb{G}$ with minimum cardinality $\kappa$. It shows that $\rho$ is not a resolving function, which is a contradiction. Therefore, $\tau$ is a minimal resolving function that attains minimum $|\tau|$ for $\mathbb{G}$. All the $R_{l}$ for $1 \leq l \leq 3$ also exhibit the intersection of cyclic order along with $\bigcap_{l=1}^{3} R_{l}=\phi$; thus, by Theorem 1, Case B, Sub case (1)a, 
assigning $\frac{1}{\kappa}$ to the vertices of $\mathbb{G}$ in $\bigcup_{t=1}^{3} R_{l}$ and calculating the summation of all the weights, we get:

$$
\operatorname{fim}(\mathbb{G})=\sum_{l=1}^{\sigma} \frac{1}{\kappa}=\frac{3}{2}
$$

\subsection{FMD of Polaroid Grid}

Theorem 3. Suppose that $\mathbb{G} \cong \mathbb{P} \mathbb{G}(m, n)$ is a polaroid grid network, taking any non-negative number $m \geq 8$ and $n \geq 4$, then $1 \leq f \operatorname{dim}(\mathbb{G}) \leq \frac{m}{4}$.

Proof. In order to prove this result, we will prove the following cases: Case I: For $m=8$ and $n=4$ :

We will make use of symmetry of the network to present the RNs for $1 \leq r \leq 4$, so the RNs for the current case are given by:

Table 9. RNs $R_{r}$ for $1 \leq l \leq 12$ and $1 \leq j \leq 4$.

\begin{tabular}{llll}
\hline RNs & Elements & RNs & Elements \\
\hline$R_{1}=R\left\{v_{1}^{1}, v_{2}^{1}\right\}$ & $V(\mathbb{G})-\{c\} \cup\left\{v_{j}^{4}, v_{j}^{5}, v_{j}^{6}, v_{j}^{7}\right\}$ & $R_{2}=R\left\{v_{2}^{1}, v_{3}^{1}\right\}$ & $V(\mathbb{G})-\{c\} \cup\left\{v_{j}^{5}, v_{j}^{6}, v_{j}^{7}, v_{j}^{8}\right\}$ \\
\hline$R_{3}=R\left\{v_{3}^{1}, v_{4}^{1}\right\}$ & $V(\mathbb{G})-\{c\} \cup\left\{v_{j}^{1}, v_{j}^{6}, v_{j}^{7}, v_{j}^{8}\right\}$ & $R_{4}=R\left\{v_{4}^{1}, v_{5}^{1}\right\}$ & $V(\mathbb{G})-\{c\} \cup\left\{v_{j}^{1}, v_{j}^{2}, v_{j}^{7}, v_{j}^{8}\right\}$ \\
\hline$R_{9}=R\left\{v_{1}^{1}, v_{1}^{3}\right\}$ & $V(\mathbb{G})-\{c\} \cup\left\{v_{j}^{2}, v_{j}^{5}, v_{j}^{6}, v_{j}^{7}\right\}$ & $R_{10}=R\left\{v_{1}^{2}, v_{1}^{4}\right\}$ & $V(\mathbb{G})-\{c\} \cup\left\{v_{j}^{3}, v_{j}^{6}, v_{j}^{7}, v_{j}^{8}\right\}$ \\
\hline$R_{11}=R\left\{v_{1}^{3}, v_{1}^{5}\right\}$ & $V(\mathbb{G})-\{c\} \cup\left\{v_{j}^{1}, v_{j}^{4}, v_{j}^{7}, v_{j}^{8}\right\}$ & $R_{12}=R\left\{v_{1}^{4}, v_{1}^{6}\right\}$ & $V(\mathbb{G})-\{c\} \cup\left\{v_{j}^{1}, v_{j}^{2}, v_{j}^{5}, v_{j}^{8}\right\}$ \\
\hline
\end{tabular}

Table 10. RNs $R_{u}$ for $1 \leq u \leq 16$ and $1 \leq j \leq 8$.

\begin{tabular}{llll}
\hline RNs & Elements & RNs & Elements \\
\hline$R\left\{v_{1}^{j}, v_{3}^{j}\right\}$ & $V(\mathbb{G})-\left\{v_{2}^{1}, v_{2}^{2}, v_{2}^{3}, v_{2}^{4}, v_{2}^{5}, v_{2}^{6}, v_{2}^{7}, v_{2}^{8}\right\}$ & $R\left\{v_{2}^{j}, v_{4}^{j}\right\}$ & $V(\mathbb{G})-\left\{v_{3}^{1}, v_{3}^{2}, v_{3}^{3}, v_{3}^{4}, v_{3}^{5}, v_{3}^{6}, v_{3}^{7}, v_{3}^{8}\right\}$ \\
\hline
\end{tabular}

Table 11. RNs $R_{u}$ for $17 \leq u \leq 20$ and $1 \leq j \leq 8$.

\begin{tabular}{llll}
\hline RNs & Elements & RNs & Elements \\
\hline$R\left\{v_{1}^{1}, v_{1}^{4}\right\}$ & $V(\mathbb{G})-\{c\} \cup\left\{v_{1}^{6}, v_{2}^{6}, v_{3}^{6}, v_{4}^{6}\right\} \cup$ & $R\left\{v_{1}^{2}, v_{1}^{5}\right\}$ & $V(\mathbb{G})-\{c\} \cup\left\{v_{1}^{7}, v_{2}^{7}, v_{3}^{7}, v_{4}^{7}\right\} \cup$ \\
& $\left\{v_{1}^{7}, v_{2}^{7}, v_{3}^{7}, v_{4}^{7}\right\}$ & & $\left\{v_{1}^{8}, v_{2}^{8}, v_{3}^{8}, v_{4}^{8}\right\}$ \\
\hline$R\left\{v_{1}^{3}, v_{1}^{6}\right\}$ & $V(\mathbb{G})-\{c\} \cup\left\{v_{1}^{1}, v_{2}^{1}, v_{3}^{1}, v_{4}^{1}\right\} \cup$ & $R\left\{v_{1}^{4}, v_{1}^{7}\right\}$ & $V(\mathbb{G})-\{c\} \cup\left\{v_{1}^{1}, v_{2}^{1}, v_{3}^{1}, v_{4}^{1}\right\} \cup$ \\
& $\left\{v_{1}^{8}, v_{2}^{8}, v_{3}^{8}, v_{4}^{8}\right\}$ & & $\left\{v_{1}^{2}, v_{2}^{2}, v_{3}^{2}, v_{4}^{2}\right\}$ \\
\hline
\end{tabular}

Table 12. RNs $R_{u}$ for $21 \leq u \leq 32$ and $2 \leq j \leq 4$.

\begin{tabular}{llll}
\hline RNs & Elements & RNs & Elements \\
\hline$R\left\{v_{j}^{1}, v_{j}^{2}\right\}$ & $V(\mathbb{G})-\{c\} \cup\left\{v_{1}^{4}, v_{1}^{5}, v_{1}^{6}, v_{1}^{7}\right\}$ & $R\left\{v_{j}^{2}, v_{j}^{3}\right\}$ & $V(\mathbb{G})-\{c\} \cup\left\{v_{1}^{5}, v_{1}^{6}, v_{1}^{7}, v_{1}^{8}\right\}$ \\
\hline$R\left\{v_{j}^{3}, v_{j}^{4}\right\}$ & $V(\mathbb{G})-\{c\} \cup\left\{v_{1}^{1}, v_{1}^{6}, v_{1}^{7}, v_{1}^{8}\right\}$ & & \\
\hline
\end{tabular}

Table 13. RNs $R_{u}$ for $33 \leq u \leq 36$ and $2 \leq j \leq 8$.

\begin{tabular}{llll}
\hline RNs & Elements & RNs & Elements \\
\hline$R\left\{v_{2}^{1}, v_{2}^{3}\right\}$ & $V(\mathbb{G})-\{c\} \cup\left\{v_{1}^{2}, v_{2}^{2}, v_{3}^{2}, v_{4}^{2}\right\} \cup R\left\{v_{2}^{2}, v_{2}^{4}\right\}$ & $V(\mathbb{G})-\{c\} \cup\left\{v_{1}^{3}, v_{2}^{3}, v_{3}^{3}, v_{4}^{3}\right\} \cup$ \\
& $\left\{v_{1}^{5}, v_{1}^{6}, v_{1}^{7}\right\}$ & & $\left\{v_{1}^{6}, v_{1}^{7}, v_{1}^{8}\right\}$ \\
\hline$R\left\{v_{2}^{3}, v_{2}^{5}\right\}$ & $V(\mathbb{G})-\{c\} \cup\left\{v_{1}^{4}, v_{2}^{4}, v_{3}^{4}, v_{4}^{4}\right\} \cup R\left\{v_{2}^{4}, v_{2}^{6}\right\}$ & $V(\mathbb{G})-\{c\} \cup\left\{v_{1}^{5}, v_{2}^{5}, v_{3}^{5}, v_{4}^{5}\right\} \cup$ \\
& $\left\{v_{1}^{1}, v_{1}^{7}, v_{1}^{8}\right\}$ & & $\left\{v_{1}^{1}, v_{1}^{2}, v_{1}^{8}\right\}$ \\
\hline
\end{tabular}


Table 14. RNs $R_{u}$ for $37 \leq u \leq 48$ and $2 \leq j \leq 4$.

\begin{tabular}{llll}
\hline RNs & Elements & RNs & Elements \\
\hline$R\left\{v_{j}^{1}, v_{j}^{4}\right\}$ & $V(\mathbb{G})-\{c\} \cup\left\{v_{1}^{6}, v_{1}^{7}\right\}$ & $R\left\{v_{j}^{2}, v_{j}^{5}\right\}$ & $V(\mathbb{G})-\{c\} \cup\left\{v_{1}^{7}, v_{1}^{8}\right\}$ \\
\hline$R\left\{v_{j}^{3}, v_{j}^{6}\right\}$ & $V(\mathbb{G})-\{c\} \cup\left\{v_{1}^{1}, v_{1}^{8}\right\}$ & $R\left\{v_{j}^{4}, v_{j}^{7}\right\}$ & $V(\mathbb{G})-\{c\} \cup\left\{v_{1}^{1}, v_{1}^{2}\right\}$ \\
\hline
\end{tabular}

Table 15. RNs $R_{u}$ for $49 \leq u \leq 60$ and $2 \leq j \leq 4$.

\begin{tabular}{llll}
\hline RNs & Elements & RNs & Elements \\
\hline$R\left\{v_{j}^{1}, v_{j}^{5}\right\}$ & $V(\mathbb{G})-\{c\} \cup\left\{v_{1}^{3}, v_{2}^{3}, v_{3}^{3}, v_{4}^{3}\right\} \cup\left\{v_{1}^{7}\right\}$ & $R\left\{v_{j}^{2}, v_{j}^{6}\right\}$ & $V(\mathbb{G})-\{c\} \cup\left\{v_{1}^{4}, v_{2}^{4}, v_{3}^{4}, v_{4}^{4}\right\} \cup\left\{v_{1}^{8}\right\}$ \\
\hline$R\left\{v_{j}^{4}, v_{j}^{8}\right\}$ & $V(\mathbb{G})-\{c\} \cup\left\{v_{1}^{2}, v_{2}^{2}, v_{3}^{2}, v_{4}^{2}\right\} \cup\left\{v_{1}^{6}\right\}$ & & \\
\hline
\end{tabular}

Table 16. RNs $R_{u}$ for $61 \leq u \leq 77$.

\begin{tabular}{|c|c|c|c|}
\hline RNs & Elements & RNs & Elements \\
\hline$R\left\{c, v_{1}^{1}\right\}$ & $\begin{array}{l}V(\mathbb{G})-\left\{v_{1}^{2}, v_{2}^{2}, v_{3}^{2}, v_{4}^{2}\right\} \\
\left\{v_{1}^{8}, v_{2}^{8}, v_{3}^{8}, v_{4}^{8}\right\}\end{array}$ & $R\left\{c, v_{1}^{2}\right\}$ & $\begin{array}{l}V(\mathbb{G})-\left\{v_{1}^{1}, v_{2}^{1}, v_{3}^{1}, v_{4}^{1}\right\} \quad \cup \\
\left\{v_{1}^{3}, v_{2}^{3}, v_{3}^{3}, v_{4}^{3}\right\}\end{array}$ \\
\hline$R\left\{c, v_{1}^{3}\right\}$ & $\begin{array}{l}V(\mathbb{G})-\left\{v_{1}^{2}, v_{2}^{2}, v_{3}^{2}, v_{4}^{2}\right\} \\
\left\{v_{1}^{4}, v_{2}^{4}, v_{3}^{4}, v_{4}^{4}\right\}\end{array}$ & $R\left\{c, v_{1}^{4}\right\}$ & $\begin{array}{l}V(\mathbb{G})-\left\{v_{1}^{3}, v_{2}^{3}, v_{3}^{3}, v_{4}^{3}\right\} \\
\left\{v_{1}^{5}, v_{2}^{5}, v_{3}^{5}, v_{4}^{5}\right\}\end{array}$ \\
\hline$R\left\{c, v_{2}^{1}\right\}$ & $\begin{array}{l}V(\mathbb{G})-\left\{v_{1}^{1}\right\} \cup\left\{v_{2}^{3}, v_{3}^{3}, v_{4}^{3}\right\} \\
\left\{v_{2}^{7}, v_{3}^{7}, v_{4}^{7}\right\}\end{array}$ & $R\left\{c, v_{2}^{2}\right\}$ & $\begin{array}{l}V(\mathbb{G})-\left\{v_{1}^{2}\right\} \cup\left\{v_{2}^{4}, v_{3}^{4}, v_{4}^{4}\right\} \cup \\
\left\{v_{2}^{8}, v_{3}^{8}, v_{4}^{8}\right\}\end{array}$ \\
\hline$R\left\{c, v_{2}^{3}\right\}$ & $\begin{array}{l}V(\mathbb{G})-\left\{v_{1}^{3}\right\} \cup\left\{v_{2}^{1}, v_{3}^{1}, v_{4}^{1}\right\} \\
\left\{v_{2}^{5}, v_{3}^{5}, v_{4}^{5}\right\}\end{array}$ & $R\left\{c, v_{2}^{4}\right\}$ & $\begin{array}{l}V(\mathbb{G})-\left\{v_{1}^{4}\right\} \cup\left\{v_{2}^{2}, v_{3}^{2}, v_{4}^{2}\right\} \cup \\
\left\{v_{2}^{6}, v_{3}^{6}, v_{4}^{6}\right\}\end{array}$ \\
\hline$R\left\{c, v_{3}^{1}\right\}$ & $V(\mathbb{G})-\left\{v_{3}^{4}, v_{4}^{4}\right\} \cup\left\{v_{3}^{6}, v_{4}^{6}\right\}$ & $R\left\{c, v_{3}^{2}\right\}$ & $V(\mathbb{G})-\left\{v_{3}^{5}, v_{4}^{5}\right\} \cup\left\{v_{3}^{7}, v_{4}^{7}\right\}$ \\
\hline$R\left\{c, v_{3}^{3}\right\}$ & $V(\mathbb{G})-\left\{v_{3}^{6}, v_{4}^{6}\right\} \cup\left\{v_{3}^{8}, v_{4}^{8}\right\}$ & $R\left\{c, v_{3}^{4}\right\}$ & $V(\mathbb{G})-\left\{v_{3}^{1}, v_{4}^{1}\right\} \cup\left\{v_{3}^{7}, v_{4}^{7}\right\}$ \\
\hline$R\left\{c, v_{4}^{1}\right\}$ & $V(\mathbb{G})-\left\{v_{2}^{1}\right\} \cup\left\{v_{4}^{5}\right\}$ & $R\left\{c, v_{4}^{2}\right\}$ & $V(\mathbb{G})-\left\{v_{2}^{2}\right\} \cup\left\{v_{4}^{6}\right\}$ \\
\hline$R\left\{c, v_{4}^{3}\right\}$ & $V(\mathbb{G})-\left\{v_{2}^{3}\right\} \cup\left\{v_{4}^{7}\right\}$ & $R\left\{c, v_{4}^{4}\right\}$ & $V(\mathbb{G})-\left\{v_{2}^{4}\right\} \cup\left\{v_{4}^{8}\right\}$ \\
\hline
\end{tabular}

Table 17. RNs $R_{u}$ for $78 \leq u \leq 81$.

\begin{tabular}{llll}
\hline RNs & Elements & RNs & Elements \\
\hline$R\left\{v_{1}^{1}, v_{1}^{5}\right\}$ & $V(\mathbb{G})-\{c\} \cup\left\{v_{1}^{3}, v_{2}^{3}, v_{3}^{3}, v_{4}^{3}\right\} \cup$ & $R\left\{v_{1}^{2}, v_{1}^{6}\right\}$ & $V(\mathbb{G})-\{c\} \cup\left\{v_{1}^{4}, v_{2}^{4}, v_{3}^{4}, v_{4}^{4}\right\} \cup$ \\
& $\left\{v_{1}^{7}, v_{2}^{7}, v_{3}^{7}, v_{4}^{7}\right\}$ & & $\left\{v_{1}^{8}, v_{2}^{8}, v_{3}^{8}, v_{4}^{8}\right\}$ \\
\hline$R\left\{v_{1}^{3}, v_{1}^{7}\right\}$ & $V(\mathbb{G})-\{c\} \cup\left\{v_{1}^{1}, v_{2}^{1}, v_{3}^{1}, v_{4}^{1}\right\} \cup R\left\{v_{1}^{4}, v_{1}^{8}\right\}$ & $V(\mathbb{G})-\{c\} \cup\left\{v_{1}^{2}, v_{2}^{2}, v_{3}^{2}, v_{4}^{2}\right\} \cup$ \\
& $\left\{v_{1}^{5}, v_{2}^{5}, v_{3}^{5}, v_{4}^{5}\right\}$ & $\left\{v_{1}^{6}, v_{2}^{6}, v_{3}^{6}, v_{4}^{6}\right\}$ \\
\hline
\end{tabular}

Table 18. RNs $\hat{R}_{u}$ for $82 \leq u \leq 89$ and $2 \leq j \leq 4$.

\begin{tabular}{llll}
\hline RNs & Elements & RNs & Elements \\
\hline$R\left\{v_{1}^{1}, v_{2}^{2}\right\}$ & $V(\mathbb{G})-\left\{v_{1}^{2}, v_{1}^{3}\right\} \cup\left\{v_{j}^{1}, v_{j}^{5}, v_{j}^{8}\right\}$ & $R\left\{v_{1}^{2}, v_{2}^{3}\right\}$ & $V(\mathbb{G})-\left\{v_{1}^{3}, v_{1}^{4}\right\} \cup\left\{v_{j}^{1}, v_{j}^{2}, v_{j}^{6}\right\}$ \\
\hline$R\left\{v_{1}^{3}, v_{2}^{4}\right\}$ & $V(\mathbb{G})-\left\{v_{1}^{4}, v_{1}^{5}\right\} \cup\left\{v_{j}^{2}, v_{j}^{3}, v_{j}^{7}\right\}$ & $R\left\{v_{1}^{4}, v_{2}^{5}\right\}$ & $V(\mathbb{G})-\left\{v_{1}^{5}, v_{1}^{6}\right\} \cup\left\{v_{j}^{3}, v_{j}^{4}, v_{j}^{8}\right\}$ \\
\hline$R\left\{v_{2}^{1}, v_{1}^{2}\right\}$ & $V(\mathbb{G})-\left\{v_{1}^{1}, v_{1}^{8}\right\} \cup\left\{v_{j}^{2}, v_{j}^{4}, v_{j}^{6}\right\}$ & $R\left\{v_{2}^{2}, v_{1}^{3}\right\}$ & $V(\mathbb{G})-\left\{v_{1}^{1}, v_{1}^{2}\right\} \cup\left\{v_{j}^{3}, v_{j}^{5}, v_{j}^{7}\right\}$ \\
\hline$R\left\{v_{2}^{3}, v_{1}^{4}\right\}$ & $V(\mathbb{G})-\left\{v_{1}^{2}, v_{1}^{3}\right\} \cup\left\{v_{j}^{4}, v_{j}^{6}, v_{j}^{8}\right\}$ & $R\left\{v_{2}^{4}, v_{1}^{5}\right\}$ & $V(\mathbb{G})-\left\{v_{1}^{3}, v_{1}^{4}\right\} \cup\left\{v_{j}^{1}, v_{j}^{5}, v_{j}^{7}\right\}$ \\
\hline
\end{tabular}


Table 19. RNs $R_{u}$ for $90 \leq u \leq 97,1 \leq j \leq 2$ and $3 \leq k \leq 4$.

\begin{tabular}{|c|c|c|c|c|c|}
\hline RNs & Elements & & & RNs & Elements \\
\hline$R\left\{v_{2}^{1}, v_{3}^{2}\right\}$ & $\begin{array}{l}V(\mathbb{G})-\left\{v_{k}^{1}, v_{k}^{8}\right\} \\
\left\{v_{j}^{2}, v_{j}^{3}, v_{j}^{4}\right\}\end{array}$ & $\cup\left\{v_{2}^{5}\right\}$ & $U$ & $R\left\{v_{2}^{2}, v_{3}^{3}\right\}$ & $\begin{array}{l}V(\mathbb{G})-\left\{v_{k}^{1}, v_{k}^{2}\right\} \quad \cup \quad\left\{v_{2}^{6}\right\} \quad \cup \\
\left\{v_{j}^{3}, v_{j}^{4}, v_{j}^{5}\right\}\end{array}$ \\
\hline$R\left\{v_{2}^{3}, v_{3}^{4}\right\}$ & $\begin{array}{l}V(\mathbb{G})-\left\{v_{k}^{2}, v_{k}^{3}\right\} \\
\left\{v_{j}^{4}, v_{j}^{5}, v_{j}^{6}\right\}\end{array}$ & $\cup\left\{v_{2}^{7}\right\}$ & $u$ & $R\left\{v_{2}^{4}, v_{3}^{5}\right\}$ & $\begin{array}{l}V(\mathbb{G})-\left\{v_{k}^{3}, v_{k}^{4}\right\} \quad \cup \quad\left\{v_{2}^{8}\right\} \quad \cup \\
\left\{v_{j}^{5}, v_{j}^{6}, v_{j}^{7}\right\}\end{array}$ \\
\hline$R\left\{v_{3}^{1}, v_{2}^{2}\right\}$ & $\begin{array}{l}V(\mathbb{G})-\left\{v_{k}^{2}, v_{k}^{3}\right\} \\
\left\{v_{j}^{1}, v_{j}^{7}, v_{j}^{8}\right\}\end{array}$ & $\cup\left\{v_{2}^{6}\right\}$ & $\cup$ & $R\left\{v_{3}^{2}, v_{2}^{3}\right\}$ & $\begin{array}{l}V(\mathbb{G})-\left\{v_{k}^{3}, v_{k}^{4}\right\} \cup\left\{v_{2}^{7}\right\} \quad \cup \\
\left\{v_{j}^{1}, v_{j}^{2}, v_{j}^{8}\right\}\end{array}$ \\
\hline$R\left\{v_{3}^{3}, v_{2}^{4}\right\}$ & $\begin{array}{l}V(\mathbb{G})-\left\{v_{k}^{4}, v_{k}^{5}\right\} \\
\left\{v_{j}^{1}, v_{j}^{2}, v_{j}^{3}\right\}\end{array}$ & $\cup\left\{v_{2}^{8}\right\}$ & $u$ & $R\left\{v_{3}^{4}, v_{2}^{5}\right\}$ & $\begin{array}{l}V(\mathbb{G})-\left\{v_{k}^{5}, v_{k}^{6}\right\} \quad \cup \quad\left\{v_{2}^{1}\right\} \quad \cup \\
\left\{v_{j}^{2}, v_{j}^{3}, v_{j}^{4}\right\}\end{array}$ \\
\hline
\end{tabular}

Table 20. RNs $\dot{R}_{u}$ for $98 \leq u \leq 1051 \leq h \leq 3$ and $2 \leq k \leq 3$

\begin{tabular}{|c|c|c|c|c|c|c|c|}
\hline RNs & Elements & & & RNs & Elements & & \\
\hline$R\left\{v_{3}^{1}, v_{4}^{2}\right\}$ & $\begin{array}{l}V(\mathbb{G})-\left\{v_{4}^{j} \mid j\right. \\
\left\{v_{h}^{2}, v_{h}^{3}, v_{k}^{4}\right\}\end{array}$ & $=$ & $1,6,7,8\} \cup$ & $R\left\{v_{3}^{2}, v_{4}^{3}\right\}$ & $\begin{array}{l}V(\mathbb{G})-\left\{v_{4}^{j} \mid j\right. \\
\left\{v_{h}^{3}, v_{h}^{4}, v_{k}^{5}\right\}\end{array}$ & $=$ & $1,2,7,8\} \cup$ \\
\hline$R\left\{v_{3}^{3}, v_{4}^{4}\right\}$ & $\begin{array}{l}V(\mathbb{G})-\left\{v_{4}^{j} \mid j\right. \\
\left\{v_{h^{\prime}}^{4} v_{h^{\prime}}^{5}, v_{k}^{6}\right\}\end{array}$ & $=$ & $1,2,3,8\} \cup$ & $R\left\{v_{3}^{4}, v_{4}^{5}\right\}$ & $\begin{array}{l}V(\mathbb{G})-\left\{v_{4}^{j} \mid j\right. \\
\left\{v_{h}^{5}, v_{h}^{6}, v_{k}^{7}\right\}\end{array}$ & $=$ & $1,2,3,4\} \cup$ \\
\hline$R\left\{v_{4}^{1}, v_{3}^{2}\right\}$ & $\begin{array}{l}V(\mathbb{G})-\left\{v_{4}^{j} \mid j\right. \\
\left\{v_{h^{\prime}}^{6} v_{h^{\prime}}^{7} v_{k}^{8}\right\}\end{array}$ & $=$ & $1,2,3,4\} \cup$ & $R\left\{v_{4}^{2}, v_{3}^{3}\right\}$ & $\begin{array}{l}V(\mathbb{G})-\left\{v_{4}^{j} \mid j\right. \\
\left\{v_{h}^{1}, v_{h}^{7}, v_{k}^{8}\right\}\end{array}$ & $=$ & $2,3,4,5\} \cup$ \\
\hline$R\left\{v_{4}^{3}, v_{3}^{4}\right\}$ & $\begin{array}{l}V(\mathbb{G})-\left\{v_{4}^{j} \mid j\right. \\
\left\{v_{h^{\prime}}^{1} v_{h^{\prime}}^{2}, v_{k}^{8}\right\}\end{array}$ & $=$ & $3,4,5,6\} \cup$ & $R\left\{v_{4}^{4}, v_{3}^{5}\right\}$ & $\begin{array}{l}V(\mathbb{G})-\left\{v_{4}^{j} \mid j\right. \\
\left\{v_{h^{\prime}}^{1} v_{h^{\prime}}^{2} v_{k}^{3}\right\}\end{array}$ & $=$ & $4,5,6,7\} \cup$ \\
\hline
\end{tabular}

Table 21. RNs $\dot{R}_{u}$ for $106 \leq u \leq 141,1 \leq j \leq 8, k=1,3,5$.

\begin{tabular}{llllll}
\hline R. N. & Elements & R. N. & Elements & R. N. & Elements \\
\hline$R\left\{v_{1}^{j}, v_{3}^{j+k}\right\}$ & $V(\mathbb{G})$ & $R\left\{v_{3}^{j}, v_{1}^{j+k}\right\}$ & $V(\mathbb{G})$ & $R\left\{v_{2}^{j}, v_{4}^{j+k}\right\}$ & $V(\mathbb{G})$ \\
\hline$R\left\{v_{4}^{j}, v_{2}^{j+k}\right\}$ & $V(\mathbb{G})$ & $R\left\{v_{1}^{j}, v_{2}^{j+k}\right\}$ & $V(\mathbb{G})$ & $R\left\{v_{2}^{j}, v_{1}^{j+k}\right\}$ & $V(\mathbb{G})$ \\
\hline$R\left\{v_{2}^{j}, v_{3}^{j+k}\right\}$ & $V(\mathbb{G})$ & $R\left\{v_{3}^{j}, v_{2}^{j+k}\right\}$ & $V(\mathbb{G})$ & $R\left\{v_{j}^{j}, v_{j+k}^{j}\right\}$ & $V(\mathbb{G})$ \\
\hline
\end{tabular}

We can see that Table 9 shows the RNs with a minimum cardinality of 16, whereas RNs with maximum cardinality have been shown in Tables 10-21, respectively. Thus, $\bigcup_{l=1}^{12} R_{l}=V(\mathbb{G})-\{c\}$. We can see that the $\left|\bigcup_{l=1}^{12} R_{l}\right|=32$ and $\left|R_{u} \cap \bigcup_{l=1}^{12} R_{l}\right| \geq\left|R_{l}\right|$, where $1 \leq u \leq 301$. Now, we define a mapping $\tau: V(\mathbb{G}) \rightarrow[0,1]$ such that $\tau\left(v_{k}^{j}\right)=\frac{1}{16}$. It is observed that $R_{l}$ for $1 \leq l \leq 12$ of $V(\mathbb{G})$ have a pairwise intersection, exhibiting a non-cyclic order with $\bigcap_{l=1}^{12} R_{l}=\phi$; therefore, by Theorem 1, Case B, Sub case (1)b, we have $1 \leq f \operatorname{dim}(\mathbb{G}) \leq \sum_{l=1}^{32} \frac{1}{16} \leq \frac{32}{16} \leq 2$

Case II: For any $m \geq 5$ and $n \geq 4$ :

Lemma 2 confirms the fact that the minimum resolving sets are $R_{1}=R\left\{v_{1}^{r}, v_{1}^{r+1}\right\}$ and $R_{2}=R\left\{v_{1}^{r}, v_{1}^{r+2}\right\}$, respectively. The same also affirms that $\left|R_{l}\right|=4 n$ and $\left|\bigcup_{l=1}^{m} R_{l}\right|=m n$ and $\left|R\{a, b\} \cap \bigcup_{l=1}^{m} R_{l}\right| \geq\left|R_{l}\right| \forall\{a, b\} \in V(\mathbb{G})$. Let $\sigma=\left|\bigcup_{l=1}^{m} R_{l}\right|=m n$ and $\kappa=\left|R_{l}\right|=4 n$. 
Then, we define a mapping $\tau: V(\mathbb{G}) \rightarrow[0,1]$, such that

$$
\tau(v)= \begin{cases}\frac{1}{\kappa} & \text { for } a \in \bigcup_{l=1}^{m} R_{l}, \\ 0 & \text { for } a \in V(\mathbb{C})-\bigcup_{l=1}^{m} R_{l} .\end{cases}
$$

It can be seen that $\tau$ is a resolving function for $\mathbb{G}$ with $n \geq 3$ because $\tau(R\{a, b\}) \geq 1$ $\forall u, v \in V(\mathbb{G})$. On the contrary, assume that there is another resolving function $\rho$, such that $\rho(u) \leq \tau(u)$ for at least one $u \in V(\mathbb{G}) \rho(u) \neq \tau(u)$. As a consequence, $\rho(R\{a, b\})<1$, where $R\{a, b\}$ is a resolving neighbourhood of $\mathbb{G}$ with minimum cardinality $\kappa$. It shows that $\rho$ is not a resolving function, which is a contradiction. Therefore, $\tau$ is a minimal resolving function that attains minimum $|\tau|$ for $\mathbb{G}$. We have seen that all the $R_{l}$ have a pairwise intersection bearing a non-cyclic order and have $\bigcap_{l=1}^{m} R_{l}=\phi$; thus, by Theorem 1 , Sub case (1)b, assigning $\frac{1}{\kappa}$ to the vertices of $\mathbb{G}$ in $\bigcup_{l=1}^{m} R_{l}$ and calculating the summation of all the weights, we get: $1 \leq f \operatorname{dim}(\mathbb{G}) \leq \sum_{l=1}^{\sigma} \frac{1}{\kappa} \leq \frac{m}{4}$.

\section{Conclusions}

In this note, we have found the sharp bounds for the FMD of connected networks. In order to test the tautology of Theorem 1, we have considered the classes of networks that are grid-like in nature-called triangular and polaroid grid networks. We end this manuscript with the following conclusions:

- The bounds lie in the interval of $\left[1, \frac{|X|}{\kappa}\right]$.

- Theorem 1 is a generalization of what was found by Liu et al. in [24] for the FMD of the generalized Jahangir Graph.

- $\quad$ The FMD of $\mathbb{T}^{n}$ is $f \operatorname{dim}\left(\mathbb{T}^{n}\right)=\frac{3}{2}$.

- The polaroid grid network has the FMD $f \operatorname{dim}(\mathbb{P G}(m, n)) \leq \frac{m}{4}$.

- It is found that the FMD of $\mathbb{P G}(m, n)$ is unbounded.

- The value of the FMD $\mathbb{T}^{n}$ is constant as well as bounded.

- Table 22 shows the summary of the results obtained for the FMD of the networks under consideration.

Table 22. Summary of obtained Results.

\begin{tabular}{llll}
\hline $\mathbb{G}$ & $f d i m$ & $\lim _{\text {dim } \rightarrow \infty}$ & Remarks \\
\hline $\mathbb{T}^{n}$ & $\frac{3}{2}$ & $\frac{3}{2}$ & Constant and Bounded \\
\hline $\mathbb{P} G(m, n)$ & $1 \leq f \operatorname{dim}(\mathbb{P} G(m, n)) \leq \frac{m}{4}$ & $\infty$ & Unbounded \\
\hline
\end{tabular}

- The behaviour of the obtained results as they tend to $\infty$ is shown graphically in Figure 4.

- Feng et al. [20] studied only the vertex transitive graphs and computed their fraction metric dimension. In this paper, the developed lower and upper bounds of FMD in Theorem 1 work for all the graphs, which may be vertex transitive and nonvertex transitive. We also illustrated the obtained results by finding the FMDs of the triangular and polaroid grids, which are non-vertex transitive graphs. 

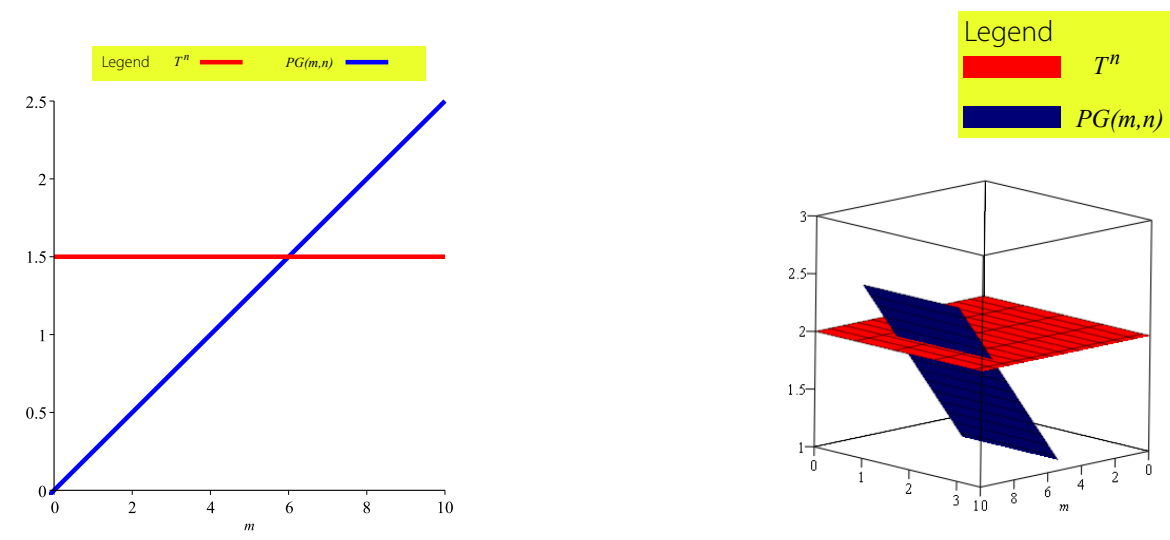

Figure 4. 2D Graphical Comparison (Left) and 3D Graphical Comparison (Right) of $f \operatorname{dim}\left(\mathbb{T}^{n}\right)$ and $\operatorname{dim}(\mathbb{P} G(m, n))$.

Author Contributions: A.H.A. contributed to the discussion of the problem, validation of results, funding and final reading; M.J. and M.K.A. contributed to the source of the problem, the collection of material, analyzing and computing the results, and wrote the paper; and A.M.A. contributed to the discussion of the problem, the methodology, and prepared the final draft. All authors have read and agreed to the published version of the manuscript.

Funding: The authors extend their appreciation to the Deanship of Scientific Research at King Khalid University for funding this work through the research groups program under grant number R.G.P.2/74/42.

Institutional Review Board Statement: Not applicable.

Informed Consent Statement: Not applicable.

Data Availability Statement: Not applicable.

Acknowledgments: The authors are very thankful for the referees' comments, which helped them to improve the results and presentation of the manuscript to a great extent.

Conflicts of Interest: The authors declare that there are no conflicts of interest regarding the publication of this paper.

\section{References}

1. Slater, P.J. Leaves of trees. Congr. Numer. 1975, 14, 549-559.

2. Melter, R.A.; Tomescu, I. Metric bases in digital geometry. Comput. Vis. Graph. Image Process. 1984, 25, 113-121. [CrossRef]

3. Khuller, S.; Raghavachari, B.; Rosenfield, A. Landmarks in graphs. Discrete Appl. Math. 1996, 70, 217-229. [CrossRef]

4. Chartrand, G.; P, Z. The theory and applicaitons of resolvability in graphs. Congr. Numer. 2003, 160, 47-68.

5. Slater, P.J. Dominating and reference sets in graphs. J. Math. Phys. Sci. 1998, 22, 445-455.

6. Buczkowski, P.; Chartrand, G.; Poisson, C.; Zhang, P. On k- dimensional graphs and their bases. Period. Math. Hung. 2003, 46, 9-15. [CrossRef]

7. Slater, P.J. Domination and location in acyclic graphs. Networks 1987, 17, 55-64. [CrossRef]

8. Harary, F.; Melter, R.A. On the metric dimension of a graph. Ars Combin. 1976, 2, 191-195.

9. Javaid, I.; Rahim, M.T.; Ali, K. Families of regular graphs with constant metric dimension. Utilitas. Math. 2008, 75, 21-33.

10. Tomescu, I.; Javaid, I. On the metric dimension of the Jahangir graph. Bull. Math. Soc. Sci. Math. Roum. 2007, 50, 371-376.

11. Imran, M.; Baig, A.Q.; Shafiq, M.K.; Tomescu, I. On metric dimension of generalized Petersen graphs $P(n, 3)$. ARS Comb. 2014, $117,113-130$.

12. Chartrand, G.; Eroh, L.; Johnson, M.; Oellermann, O.R. Resolvability in graphs and the metric dimension of a graph. Discrete Appl. Math. 2000, 105, 99-113. [CrossRef]

13. Imran, M.; Siddiqui, M.K.; Naeem, R. On metric dimension of generalized Petersen Multigraphs. IEEE Access 2018, 6, 74328-74338. [CrossRef]

14. Chu, Y.M.; Nadeem, M.F.; Azeem, M.; Siddiqui, M.K. On sharp bounds on partition dimension of convex polytopes. IEEE Acces 2020, 8, 224781-224790. [CrossRef]

15. Liu, J.B.; Nadeem, F.M.; Azeem, M. Bounds on the partition dimension of convex polytopes. Comb. Chem. High Throughput Screen. 2020. [CrossRef] 
16. Nadeem, M.F.; Azeem, M.; Khalil, A. The locating number of hexagonal M obius ladder network. J. Appl. Math. Comput. 2021, 66, 149-165. [CrossRef]

17. Currie, J.; Oellermann, O.R. The metric dimension and metric independence of a graph. J. Combin. Math. Combin. Comput. 2001, 39, 157-167.

18. Arumugam, S.; Mathew, V.; Shen, J. The fractional metric dimension of graphs. Discret. Math. 2012, 312, 1584-1590. [CrossRef]

19. Arumugam, S.; Mathew, V.; Shen, J. On fractional metric dimension of graphs. Discret. Math. Algorithms Appl. 2013, 5, 1-8. [CrossRef]

20. Feng, M.; Lv, B.; Wang, K. On the fractional metric dimension of graphs. Discret. Appl. Math. 2014, 170, 55-63. [CrossRef]

21. Feng, M.; Wang, K. On the metric dimension and fractional metric dimension of the hierarchical product of graphs. Appl. Anal. Discrete Math. 2013, 7, 302-313. [CrossRef]

22. Saputro, S.W.; Semanicova-Fenovc, A.; Baca, M.; Lascsakova, M. On fractional metric dimension of comb product graphs. Stat. Optim. Inf. Comput. 2018, 6, 150-158. [CrossRef]

23. Feng, M.; Wang, K. On the fractional metric dimension of corona product graphs and lexicographic product graphs. arXiv 2012, arXiv:1206.1906v1.

24. Liu, J.B.; Kashif, A.; Rashid, T.; Javaid, M. Fractional metric dimension of Generalized Jahangir Graph. Mathematics 2019, 7, 100. [CrossRef] 